\title{
L'estimation kinesthésique des distances : études comportementales et analyse probabiliste
}

\author{
Edouard Gentaz, Henry Faineteau, Estelle Gilet, Jéremy Bluteau, \\ Richard Palluel-Germain \& Julien Diard \\ CNRS et Université de Grenoble (UPMF), France
}

Titre courant : estimer des distances avec la main

Correspondance : Dr. Edouard Gentaz, CNRS, Laboratoire de Psychologie et Neurocognition, SHS, Domaine Universitaire, Université Pierre Mendès France (Grenoble 2), 1251 avenue centrale, BP 47, 38040 Grenoble Cedex 9, France

E-mail : Edouard.Gentaz@upmf-grenoble.fr 


\section{Résumé}

Cette revue critique concerne les processus psychologiques par lesquels nous estimons des distances en utilisant les indices kinesthésiques en l'absence d'informations visuelles. Le paradigme expérimental utilisé par les chercheurs consiste à demander à des sujets (sous occlusion visuelle momentanée) d'explorer avec le système bras-main une distance cible et puis de la reproduire avec la même main. L'analyse des données de la littérature scientifique montre que l'estimation kinesthésique des distances dépend de trois types de facteurs : 1) les indices géométriques, 2) les indices de force et 3) les facteurs cognitifs. De plus, l'analyse des mesures utilisées par les chercheurs pour quantifier cette estimation révèle que les différents types de mesure -l'erreur constante, l'erreur variable et l'erreur absolue- sont traités soit conjointement soit indépendamment. En replaçant ces mesures dans le cadre d'une analyse probabiliste, il apparaît qu'aucune de ces dernières n'est intrinsèquement meilleure que les autres. En réalité, elles font des hypothèses implicites différentes. Discuter de la pertinence de ces mesures d'erreurs revient donc à discuter de la validité de ces hypothèses sous-jacentes, ce qui, heureusement, peut être examiné expérimentalement. 


\begin{abstract}
The purpose of the present paper is to review studies examining the estimation of distances on the basis of kinesthetic cues. In this kind of task, blindfolded participant explores a path (target distance) with one arm-hand system and reproduces later its distance with the same arm-hand system. The analysis of data shows the estimations depend on three types of factor : 1) the geometrical cues ; 2) the force cues ; and 3) the cognitive factors. Moreover, the analysis about the different variables used by researchers to measure the performance shows that the different types of errors - constant, absolute and variable errors- have been processed either conjointly or independently. By casting these error measures in the probabilistic modeling framework, we show that none of these is inherently more adequate than the others; they differ in the underlying, implicit hypotheses they assume. Discussing the relevance of these error measures thus amounts to discussing the validity of these hypotheses, which fortunately, could be assessed experimentally.
\end{abstract}




\section{Introduction}

Cette revue critique concerne les processus psychologiques par lesquels nous estimons des distances en utilisant les indices kinesthésiques, en l'absence d'informations visuelles. Ce thème engendre immédiatement une question classique : l'espace représenté de manière interne a-t-il les mêmes propriétés que l'espace physique externe ? Plusieurs études répondent par la négative. Ainsi, la perception haptique (tactilo-kinesthésique) de différentes propriétés géométriques est systématiquement déviée de la réalité physique. Par exemple, Kappers (1999, 2002) et Kappers et Koenderink (1999) montrent que les adultes (travaillant sous occlusion visuelle momentanée) sont incapables, avec leurs mains, de positionner parallèlement dans l'espace physique deux barres pouvant tourner sur $360^{\circ}$ dans un plan (sagittal, horizontal ou frontal). Les sujets estiment que les deux baguettes sont parallèles lorsqu'elles dévient de plusieurs dizaines de degrés angulaires. Ces déviations systématiques sont aussi observées en vision (Cuijpers, Kappers et al., 2000 ; Cuijpers, Kappers et al., 2002). Ainsi, deux barres (séparées par un angle visuel de $60^{\circ}$ du point de vue de l'observateur) doivent différer de $20^{\circ}$ (en orientation) pour être estimées comme parallèles par les sujets. Ces résultats confirment cette ancienne idée (Blumenfeld, 1937) que l'espace visuel représenté mentalement n'est pas euclidien (cf. Gentaz et Hatwell, 2008). Ces résultats suggèrent que l'espace représenté, perçu par le sens kinesthésique, ne devrait pas être non plus euclidien. Dans ce cas, l'estimation kinesthésique des distances ne devrait pas seulement dépendre de la géométrie des stimuli, comme la longueur physique des segments. Avant de présenter les autres facteurs qui contribuent à ces estimations, revenons brièvement au sens kinesthésique.

Bien que le concept de kinesthésie soit encore débattu dans la littérature, nous utiliserons ici ce terme pour faire référence au sens de la position et du mouvement des membres (Kandel et Schwartz, 1991 ; Gandevia et Burke, 1992 ; Roll, 1994 ; Gandevia, 1996). Les caractéristiques fonctionnelles du sens kinesthésique et des processus sous-jacents 
sont encore assez mal connues pour plusieurs raisons (cf. Gentaz, Hatwell et al., 2006). La première raison est que le sens kinesthésique trouve son origine dans l'activité de multiples mécanorécepteurs (les fuseaux neuromusculaires ; les organes tendineux de Golgi ; les récepteurs articulaires ; et les récepteurs cutanés ; pour une discussion sur les contributions respectives de chacun, cf. Clark \& Horch, 1986 ; Matthews, 1988 ; Proske, Wise et al., 2000 ; Proske, 2005) qui interagissent de manière complexe avec l'environnement. La deuxième raison est que les contractions musculaires génèrent des tensions dans l'ensemble des tissus dans lesquels il y a des mécanorécepteurs cutanés et proprioceptifs. L'activité des mécanorécepteurs dépend de forces externes comme la gravité ou des forces de pression, mais aussi de forces internes qui ne sont pas directement observables. Ces forces internes peuvent exister même en l'absence de la production de mouvement (par exemple pendant la contraction de muscles antagonistes). Une description complète du stimulus nécessiterait une caractérisation de la façon dont les forces internes et externes sont distribuées sur le corps. La troisième raison est que la plupart du temps, ces processus opèrent de manière autonome, à cause du caractère inconscient des entrées proprioceptives. Malgré ces caractéristiques intrinsèques à ce sens (qui sont aussi valables pour le sens haptique), nous allons discuter des principaux résultats observés dans la littérature depuis le début des années soixante-dix. Nous allons voir aussi que dans ces études sur l'estimation des distances, il est parfois difficile de faire une distinction claire entre le sens kinesthésique et le sens haptique (tactilokinesthésique). En effet, dans de nombreuses études, les deux sens se fondent principalement sur des informations issues des mouvements du système bras-main et peu sur des informations cutanées.

Ainsi, l'objectif de cette revue est de montrer que la perception kinesthésique des distances dépend, d'une part, d'indices de géométrie et de force fortement corrélés dans des situations « naturelles ou écologiques » (cf. Robles-de-la-Torres et Hayward, 2001) et, d'autre 
part, de facteurs cognitifs. L'examen des effets de ces différents facteurs sur les performances des sujets (et leurs patterns d'erreur) va ainsi montrer que l'humain ne se représente pas mentalement les distances, perçues via un mouvement de la main, dans un espace euclidien. Pour cela, nous allons présenter les facteurs qui affectent la perception kinesthésique des distances, classés en trois groupes distincts : 1) les indices géométriques ; 2) les indices de force et 3) les facteurs cognitifs. Ensuite, nous discuterons des implications théoriques, du point de vue d'une probabiliste, des différentes mesures utilisées dans les études présentées.

\section{La contribution des indices géométriques}

Pour étudier l'estimation kinesthésique des distances, les chercheurs entre les années 60 et 80 ont souvent utilisé le paradigme expérimental de positionnement de levier. Un sujet, yeux bandés, est situé face à un appareillage contenant un levier, fixé à une sorte de rail, et qui peut être déplacé horizontalement ou verticalement. Ainsi, le mouvement est limité à une seule dimension de l'espace. Une cheville est placée à une certaine distance dans le rail. Lorsque le mouvement cible atteint l'arrivée, la cheville est ôtée, et le sujet doit produire un mouvement en réponse durant lequel il a pour tâche de reproduire librement soit:1) la position de l'arrivée (le point de départ est alors modifié pour empêcher le sujet d'utiliser l'indice de distance). Dans cette condition, la position de la main est le seul indice restant disponible ; 2) la distance entre le point de départ et le point d'arrivée (le point de départ est alors modifié pour empêcher le sujet d'utiliser l'indice de localisation. Dans cette condition, le sujet doit reproduire la longueur du mouvement cible en se basant uniquement sur l'indice de distance ; 3) à la fois la position et la distance (reproduction de la même distance, le point de départ reste le même). Ainsi, pour reproduire le mouvement cible, le sujet peut utiliser à la fois les indices de position et de distance. 


\subsection{Effet de la longueur des mouvements}

Il est souvent observé que les distances longues sont sous-estimées et que les distances courtes sont surestimées (e.g., Adams et Dijkstra, 1966 ; Keele et Ells, 1972 ; Marteniuk et Roy, 1972 ; Marteniuk, Shields et al., 1972 ; Diewert, 1975 ; Marteniuk, 1976 ; Hall et Wilberg, 1977 ; Kelso, 1977b). Wilberg et Girouard (1976) nomment ce phénomène « effet de l'étendue » (traduit de l'anglais « range effect»). Ils le définissent comme le mécanisme par lequel les sujets s'adaptent à une série de mouvements qu'ils essaient de reproduire, soit en progressant ou soit en régressant vers la moyenne de cette série de mouvements. Stelmach (1973) et Wilberg et Girouard (1976) observent une tendance opposée, avec des sujets qui surestiment les mouvements longs et sous-estiment les mouvements courts. Nous verrons par la suite que cette contradiction peut être expliquée par des différences entre les conditions expérimentales proposées et le type des erreurs mesurées.

Ces différences d'estimation entre trajets courts et trajets longs sont également observées dans des tâches où un délai ou une tâche cognitive sont introduits avant la tâche d'estimation. Ainsi, Keele (1968) observe que l'effet du délai est plus marqué pour les mouvements longs que pour les mouvements courts. Inversement, il remarque que l'effet d'une tâche cognitive, avant l'exécution du mouvement réponse, est plus marqué pour les mouvements courts que pour les mouvements longs. Ce type d'interaction conduit Keele à suggérer l'existence de différents systèmes mnésiques impliqués dans la rétention des mouvements courts et celle des mouvements longs. De manière similaire, Kelso (1977b) suggère une différence dans le traitement des mouvements courts par rapport à celui des mouvements longs : la reproduction des mouvements courts serait plus susceptible d'être perturbée que celle des mouvements longs. L'effet d'une tâche cognitive durant l'intervalle n'agirait que sur les mouvements courts $(0-20 \mathrm{~cm})$. Kelso ajoute que ces derniers requièrent un traitement plus important que les mouvements moyens ou longs $(20-60 \mathrm{~cm})$. Au contraire, 
d'autres études montrent (en utilisant d'autres types d'erreurs, cf. plus bas) que plus la longueur du mouvement est importante, plus les erreurs augmentent (e.g. Keele et Ells, 1972 ; (Posner 1967 ; Pepper et Herman, 1970 ; Stelmach et Bassin, 1971 ; Stelmach, 1973). Lorsque des sujets doivent estimer de manière kinesthésique la distance d'un trajet, ils disposent des deux indices : la position finale du trajet préalablement testé et/ou la distance du trajet effectué. L'utilisation de ces indices n'est pas exclusif (Keele, 1968). Il est intéressant de noter que la prise en compte et l'utilisation de différents indices seraient affectés en fonction de la longueur de la distance cible. L'indice de localisation serait utilisé pour la reproduction des mouvements longs alors que, pour les mouvements plus courts, il semblerait que l'indice de distance fournisse des informations pertinentes (Gundry ,1975; Keele et Ells, 1972 ; Marteniuk et Roy, 1972 ; Marteniuk et al., 1972 ; Roy, 1977 ; Stelmach et Wilson, 1970 ; Stelmach, Kelso et al., 1975 ; Wrisberg et Winter, 1985). Les deux types d'indices combinés auraient alors un effet différent en fonction de la longueur du mouvement à effectuer par le sujet.

De plus, Gundry (1975) observe que lorsqu'un sujet a pour tâche de reproduire la localisation, si le point de départ du mouvement est modifié (c'est-à-dire si l'indice de distance n'est plus disponible), alors une erreur de distance intervient dans la reproduction des mouvements courts mais pas pour celle des mouvements longs. D'autre part, Keele et Ells (1972) observent que pour les mouvements les plus longs, les sujets reproduisent aussi bien la localisation du point d'arrivée du mouvement cible lorsque les indices de localisation et de distance sont conjointement disponibles que lorsque seul l'indice de localisation est disponible. Par contre, quand les mouvements sont courts (mouvements impliquant une variation angulaire de l'articulation de l'épaule de moins de $30^{\circ}$ ), la reproduction de la localisation est nettement plus précise lorsque le sujet bénéficie des deux types d'indice que lorsqu'il se base uniquement sur l'indice de localisation. Quand l'amplitude du mouvement 
cible est importante, alors l'indice de localisation qui en résulte serait nettement moins affecté par les changements de point de départ du mouvement réponse que lorsque l'amplitude est faible. Selon Smyth (1984), pour les mouvements à forte amplitude, les indices de localisation sont faciles à discriminer, et peuvent ainsi être traités globalement, à l'intérieur d'un système de référence. Lorsque les mouvements sont de faible amplitude, il serait plus difficile de distinguer différentes positions dans l'espace, et l'indice de distance prendrait alors plus d'importance.

Enfin, il est important de noter qu'aucun consensus n'a été trouvé pour distinguer un mouvement long d'un mouvement court. Les distances peuvent varier de $5 \mathrm{~cm}$ (Johnson et Simmons, 1980) à $68 \mathrm{~cm}$ (Toole, Christian et al., 1982). Les mouvements peuvent couvrir des secteurs angulaires allant de $10^{\circ}$ (Laabs, 1973) à $140^{\circ}$ (Stelmach et Wilson, 1970). On remarque également que dans les recherches faites par un même auteur, on peut observer des variations. Par exemple, Laabs en 1974, utilise des mouvements couvrant des angles de $20^{\circ}$, $25^{\circ}$ et $30^{\circ}$ comme mouvements courts et de $40^{\circ}, 45^{\circ}$ et $50^{\circ}$ comme mouvements longs, alors qu'en 1976, le même auteur compare des mouvements courts variant de $35^{\circ}$ à $45^{\circ}$ (considérés comme longs en 1974!) à des mouvements longs de $50^{\circ}-60^{\circ}$. La distinction entre mouvements longs et mouvements courts demeure problématique. Cette dichotomie artificielle rend alors difficile la synthèse des travaux effectués jusqu'à présent.

\subsection{Effet de l'indice à estimer : distance ou localisation}

Comme indiqué plus haut, Laabs (1973) est le premier à distinguer expérimentalement le codage des indices kinesthésiques de celui des indices de localisation sous occlusion visuelle momentanée. Pour en arriver là, il fait varier les indices de distance et de localisation (point d'arrivée du mouvement) de manière indépendante. Dans son expérience, il utilise un rail curviligne sur lequel les sujets déplacent un levier jusqu'à une butée afin d'effectuer le mouvement cible. L'arc dessiné par le rail était divisé en trois secteurs de 20 degrés dans 
lesquels pouvait se situer le point d'arrivée du mouvement cible. Afin de tester la mémoire de l'étendue du mouvement, lors de la phase de réponse, le mouvement réponse commençait à un endroit différent de celui fixé lors de la présentation. Afin de tester la rétention de l'indice de localisation (concernant le point d'arrivée du mouvement), le sujet devait, lors de la phase test, indiquer à nouveau le point d'arrivée alors que l'étendue du mouvement à effectuer était modifiée. Dans la condition où l'indice de localisation devait être reproduit, les performances étaient nettement meilleures que dans la condition où c'est l'indice de distance qui devait être reproduit. La conclusion de Laabs était la suivante : "distance information appears to spontaneously decay, while location information seems rehearsable in some manner as long as processing capacity is available »(Laabs, 1973, p. 175). Cependant, Laabs (1973) observa des erreurs similaires dans les deux conditions lorsque le rappel se faisait de manière immédiate. La supériorité de la précision de la reproduction de la localisation apparaît si un délai d'une vingtaine de secondes sépare la phase de présentation de celle du rappel. Ces résultats mettent en évidence la grande labilité des indices de distance par rapport à ceux de localisation.

Si on accepte que ces deux aspects du mouvement peuvent êtres séparés, alors pour certains auteurs, le codage de l'indice de localisation est meilleur que celui de la distance (Diewert, 1975; Keele et Elis, 1972 ; Marteniuk, 1973 ; Stelmach, Kelso et al., 1975 ; Laabs et Simmons, 1981). Ces derniers auteurs affirment que la localisation du point d'arrivée du mouvement serait codée de manière centrale, dans une forme stable, et résistante à l'oubli (Keele et Ells, 1972 ; Marteniuk et Roy, 1972 ; Laabs, 1973 ; Diewert, 1975 ; Roy 1977). En ce qui concerne l'information de distance, les auteurs ne la considèrent généralement pas comme une source fiable dans le codage, la mémorisation et la reproduction du mouvement. Les informations de distance s'effaceraient progressivement avec le temps et ne pourraient pas être répétées mentalement (Adams et Dijkstra, 1966 ; Posner, 1967 ; Williams, Beaver et al. 
1969). Pour d'autres auteurs, au contraire, la distance pourrait être codée de même manière que la localisation (Marteniuk, 1973 ; Walsh et Russel, 1979). Pour Marteniuk (1973), la phase de codage du mouvement cible pourrait impliquer un codage de l'information de distance. Ainsi les informations de distance et de localisation seraient représentées de manière centrale (cf. aussi Jones, 1974 ; Stelmach, Kelso et al., 1975 ; Roy, 1976 ; Roy, 1977 ; Roy, 1978). Pour Hagman et Francis (1975), la différence entre distance et localisation serait due à des processus attentionnels. Lorsque le sujet sait quel indice il devra reproduire, alors la reproduction des indices de distance et celle des indices de localisation deviennent comparables. Enfin, d'après les données de Walsh, Russel et al. (1979), le rappel de l'information de distance est aussi bien effectué que celui de l'information de localisation, quelque soit le mouvement.

Les recherches de Hermelin et O'Connor (1975) chez les enfants alimentent la discussion concernant la différence de codage des distances et des localisations. En effet, ils observent que les enfants aveugles jugent avec plus de précision les distances que les localisations bien que la plupart des données montrent que les sujets, enfants ou adultes, nonvoyants ou privés de vision, codent les localisations de manière plus précise que les distances (Colley et Colley, 1981 ; Laabs et Simmons, 1981). Cette discordance entre les résultats de ces recherches semble due à la nature de la distance cible. Alors que la plupart des recherches précédentes impliquent des mouvements latéraux (coupant perpendiculairement l'axe médiosagittal), Hermelin et O'Connor (1975) imposent à leurs sujets des mouvements verticaux, effectués sur un plan frontal, et par conséquent alignés sur l'axe médio-sagittal. Ainsi, le codage spatial des distances pourrait se faire selon un système de référence égocentré (fondé sur le corps), expliquant ainsi les bonnes performances des enfants.

En dépit de ces résultats contradictoires, les revues semblent s'accorder sur le fait que la capacité à estimer une localisation est meilleure que celle à estimer une distance (cf. aussi, 
Rosembaum, Loukolopoulos et al., 1995). Par conséquent, si la distance et la localisation sont codées de manières différentes, les signaux kinesthésiques générés par ces deux tâches doivent différer. Au niveau psychologique, l'interprétation de ces résultats avancée le plus souvent est que le rappel de la distance du mouvement est variable et moins précise parce qu'elle dépend principalement de la mémoire des inputs kinesthésiques, et que le rappel de l'indice de localisation est précis car il serait codé par rapport à un système de référence égocentré (Russel, 1976 ; Millar, 1994). Au niveau neurophysiologique, quelques auteurs (e.g., Adams, 1977) ont suggéré que les récepteurs articulaires auraient une importance particulière lors du codage de la localisation, tandis que d'autres récepteurs seraient responsables du codage de la distance. Cette hypothèse semble difficilement soutenable. En effet, la position de l'articulation et la longueur du muscle d'un même segment corporel sont directement reliées ; l'angle d'une articulation ne peut pas changer de manière indépendante de la longueur des muscles. Ainsi quand la position d'une articulation est modifiée, l'ensemble des récepteurs kinesthésiques est affecté. De plus, une telle supposition n'est pas soutenue par les données de la neurophysiologie. Il n'existe pas de mécanisme qui ferait intervenir différents types de récepteurs proprioceptifs en fonction du but du mouvement, - bouger à un certain endroit ou une certaine distance. McCloskey (1977) suggère que ce sont principalement les afférences musculaires qui sont responsables du sens du mouvement et de la position, bien que les récepteurs tendineux, articulaires et cutanés fournissent tous ensemble des sources multiples de stimulations kinesthésiques et dans la plupart des cas, ils (mouvement et position) sont vraisemblablement traités ensemble par le système nerveux central.

\subsection{Effet de l'interaction des deux indices -distance et localisation-à estimer}

L'étude de l'interférence entre les informations de position et de distance initialement entreprise par Kerr (1978), Walsh et Russel (1979, 1980), Walsh (1981a) et Walsh, Russel et 
al., (1981b), décrite plus haut, a été ensuite analysée en détails par Imanaka et Abernethy (Imanaka, 1989 ; Imanaka et Abernethy, 1992, 1992b, 2000 ; Imanaka, Nishihira et al., 1996), qui ont tenté d'en isoler les sources. Lors de la perception/reproduction de la localisation du point d'arrivée, les sujets ont tendance à sous-estimer l'éloignement de la localisation du point d'arrivée lorsque le point de départ du mouvement réponse est éloigné de la cible, et à la surestimer lorsqu'il est proche. Par contre, on retrouve le pattern inverse lorsque la tâche consiste à reproduire la distance du mouvement cible : les sujets ont tendance à surestimer la distance lorsque le point de départ du mouvement réponse est éloigné de la cible, et à la sousestimer lorsqu'il est proche (Figure 1).

\section{(insérer Figure 1)}

Le phénomène d'interférence entre distance et localisation est un effet robuste pour les mouvements du bras et qui persiste pour n'importe quel type de mouvement (contraint ou présélectionné ; Walsh, Russel et al., 1979), avec des intervalles de différentes durées (Walsh et Russel, 1979), avec ou sans activité durant l'intervalle (Walsh et al., 1981b), et également entre des groupes de sujets dont les capacités d'imagerie mentale diffèrent (Walsh et al., 1980). Le seul facteur qui semble modifier de manière systématique l'effet d'interférence distance-localisation est la longueur du mouvement cible. Quand la longueur du mouvement est de $16 \mathrm{~cm}$ ou moins, la reproduction de la distance du mouvement ne semble pas être affectée par les changements de point de départ (Walsh, 1981a). Par contre, la reproduction de la localisation du point final du mouvement cible n'est pas influencée par les changements de point de départ à partir du moment où le mouvement cible excède $40 \mathrm{~cm}$ (Wrisberg et Winter, 1985 ; Wrisberg, Millslagle et al., 1987). 
Pour Imanaka et Abernethy (2000), ce phénomène serait la manifestation d'une interférence entre des traitements conscients et inconscients. Le phénomène d'interférence dans le rappel à court terme d'un mouvement du bras serait l'expression d'une interaction entre les représentations explicites et implicites disponibles lors du mouvement cible et du mouvement réponse. Dans la tâche utilisée, lors de la présentation du mouvement cible, le sujet se concentre généralement soit sur l'indice de distance, soit sur celui de localisation, et réutiliserait cette information lors du mouvement réponse. D'autre part, cette interférence mutuelle entre les représentations sensori-motrices explicites (conscientes) et implicites (inconscientes) peut s'atténuer jusqu'à disparaître lorsque les sujets doivent reproduire les deux indices (Imanaka et Abernethy, 1992a). Les auteurs suggèrent ainsi que, dans ce cas, les indices non spécifiques du mouvement sont traités et formés en une représentation explicite, éliminant ainsi toute interférence mutuelle possible entre les représentations implicites et explicites (Imanaka et Abernethy, 2000).

\subsection{Effet des distances sinueuses}

Dans toutes les études précédentes, les distances perçues et reproduites sont des segments. Cependant, il existe des recherches récentes qui examinent comment des adultes (travaillant sous occlusion visuelle momentanée) infèrent la distance euclidienne (DE) entre les points de départ et d'arrivée d'un trajet curviligne situé dans un espace bidimensionnel (i.e., la ligne droite reliant le point de départ et d'arrivée) avec leurs mains. Cette question de l'intégration de trajet fut abordée en premier lieu par Lederman, Klatzky et al. (1985). Dans cette expérience, des sujets voyants devaient explorer avec l'index droit des trajets curvilignes composés de points en relief. Plusieurs distances euclidiennes (DE) ont été testées (de 2,5 à $15,2 \mathrm{~cm}$ ) avec pour chacune d'elles des détours sinueux 2, 4, 6 et 8 fois plus longs. Les résultats révèlent tout d'abord une erreur d'estimation systématique après l'exploration manuelle des trajets rectilignes. Ensuite, l'analyse des estimations des inférences des DE 
montre une augmentation des erreurs au fur et à mesure que la longueur des trajets sinueux à explorer augmente. Cette différence entre les erreurs de DE produites après les trajets curvilignes et celles après le trajet rectiligne correspondant se nomme «l'effet du détour ». Cet effet du détour est observé dans les mêmes conditions chez des aveugles complets de naissance et avec d'autres types de trajets formés de deux segments (Lederman, Klatzky et al., 1987). Selon les auteurs, les sujets mettraient en œuvre des processus fondés sur un codage de la distance par la dynamique même du mouvement d'exploration manuelle (cf. aussi Millar, 1994). En d'autres mots, la distance semble codée, au moins en partie, par une séquence de mouvements. Ce mode de codage serait à l'œuvre indépendamment de l'expérience visuelle des sujets et seulement lorsque le système épaule-main des adultes serait largement engagé au cours de l'exploration. Un mode de codage similaire serait aussi à l'œuvre pour les tâches où les sujets voyants (adultes et enfants) ou aveugles doivent inférer la localisation spatiale d'un point à partir d'une exploration manuelle (Gentaz et Gaunet, 2006 ; Gaunet \& Gentaz, sous presse).

Il a été remarqué par Faineteau, Gentaz et Viviani (2003, 2005) que le type d'exploration (chemins en braille) et le type de tâche utilisés par l'équipe de Lederman était très coûteux sur le plan attentionnel. En effet, à tout moment, le sujet doit trouver son chemin et son mouvement d'exploration peut être orienté hors du trajet. Par ailleurs, il a la possibilité de rebrousser chemin, accentuant ainsi le caractère séquentiel et successif de l'exploration. Enfin, les différents trajets sinueux proposés par les auteurs ne sont pas décrits mathématiquement. Pour remédier à ces deux soucis expérimentaux, Faineteau et al. $(2003,2005)$ ont donc étudié l'inférence de la DE à l'aide d'un système permettant aux sujets d'explorer des trajets, rectilignes ou curvilignes (définis mathématiquement) prédéfinis sur le plan horizontal, à l'aide d'un stylet introduit dans le sillon d'une plaque gravée, permettant ainsi de mieux contrôler la nature du trajet exploré. Lors de la phase de 
réponse, à l'aide d'un mouvement rectiligne, les sujets devaient inférer la DE séparant les points de départ et d'arrivée du trajet qui venait d'être exploré à l'aide du stylet (Figure 2). Ces études montrent que des trajets de petite échelle (couvrant une portion réduite de l'espace d'action) impliquent un accroissement des erreurs d'estimation de la DE (constamment surestimée) en fonction de la longueur du détour. Au contraire cet effet de détour n'est pas observé pour des trajets de plus grande échelle. Dans ce cas, l'estimation de la DE (constamment sous estimée) n'est pas influencée par la longueur du trajet.

(insérer Figure 2)

Cependant, utilisant les mêmes longueurs, Faineteau et al. (2005) remarquent dans une autre expérience que cet effet du détour n'est pas présent dans le cas de trajets semielliptiques où aucun point d'inflexion n'est, par définition, présent, et ce quelle que soit la taille de l'espace de travail (Figure 3). Par ailleurs, l'analyse des temps du mouvement dans ces différentes expériences montre que les erreurs d'estimation de DE ne sont pas liées à la vitesse du mouvement d'encodage.

(insérer Figure 3)

L'analyse des mouvements des sujets permet de mieux expliquer la présence de l'effet du détour observé dans l'expérience de Faineteau et al. (2003). En effet, il faut noter que tous les trajets stimuli (curvilignes) et les trajets réponses (rectilignes) se situaient dans le plan horizontal des sujets et que, par conséquent, les mouvements manuels s'effectuaient selon une direction globalement sagittale, successivement centripète et centrifuge ou vice versa. Ainsi, lorsque le point de départ est éloigné par rapport au tronc du 
sujet, le mouvement manuel est dirigé vers le corps pour la phase d'encodage et dans la direction inverse pour la phase de réponse. La dynamique est inverse lorsque le point de départ se situe proche par rapport au tronc du sujet. Selon Faineteau et al. (2003, 2005), les erreurs d'estimation proviendraient ainsi de la difficulté à dissocier les composantes sagittale (pertinente pour l'estimation de la DE) et orthogonale (non pertinente) du mouvement précédemment effectué. Cette difficulté serait reliée à la fois à la taille du trajet et à la présence de points d'inflexion. En effet, leur présence dans une partie réduite de l'espace implique des changements à la fois géométriques (le signe de la courbure le long de la trajectoire) mais également biomécanique (la synergie des muscles impliquée dans le système bras-main). Avec la présence de points d'inflexion et des changements géométriques et biomécaniques qu'ils engendrent, cette décomposition serait plus difficile pour les trajets de petites échelles, les points d'inflexion étant davantage rapprochés dans l'espace. L'effet de détour pourrait être expliqué plutôt par la présence de points d'inflexion le long du trajet curviligne que par sa longueur per se.

Pour montrer que cet effet de détour est expliqué plutôt par la présence de points d'inflexion le long du trajet curviligne que par l'augmentation de sa longueur, Faineteau, Palluel-Germain et al. (2007) testent deux types de trajets à explorer (Figure 4). Dans le premier type (échelle réduite), la DE est de 7,5 cm et les trajets curvilignes ont une longueur fixe de $15 \mathrm{~cm}(2 \mathrm{xDE})$ dans lesquels le nombre de points d'inflexion varie $(2,4$ ou 6). Dans le deuxième type (échelle importante), la DE est de $22,5 \mathrm{~cm}$ et les trajets curvilignes ont une longueur fixe de $45 \mathrm{~cm}(2 x D E)$ dans lesquels le nombre de points d'inflexion varie aussi $(2,4$ ou 6$)$. Les résultats confirment que les estimations de la DE sont similaires (pas d'effet du détour) après les trajets explorés dans la région importante de l'espace de travail $\left(\mathrm{I}_{0}, \mathrm{I}_{2}, \mathrm{I}_{4}\right.$ et $\left.\mathrm{I}_{6}\right)$, En revanche, pour les trajets explorés dans la région réduite de l'espace de travail, i.e., les trajets de l'échelle réduite, un effet de détour est observé 
puisqu'il existe une augmentation de l'erreur des estimations de la DE après les trois trajets curvilignes (deux fois plus longs) par rapport à la simple reproduction du segment $\left(\mathrm{R}_{0}\right)$. Cependant, l'amplitude de ces erreurs n'est pas significativement différente entre les trajets curvilignes $\left(\mathrm{R}_{2}, \mathrm{R}_{4}\right.$ et $\left.\mathrm{R}_{6}\right)$ comprenant un nombre différent de point d'inflexion. En d'autres termes, l'amplitude des erreurs observée dans les trajets de petites échelles, bien qu'elle augmente, ne semble pas être affectée significativement par la présence de 2, 4 ou 6 points d'inflexion. Ainsi, c'est bien la présence de points d'inflexion qui est responsable de l'effet du détour, et ceci quel que soit le nombre de ces points d'inflexion.

En conclusion, l'ensemble de ces résultats suggère que l'effet de détour observé dans des études précédentes n'est pas directement lié à l'augmentation de la longueur des trajets curvilignes mais plutôt à la présence de points d'inflexion dans une partie restreinte de l'espace de travail. En d'autre terme, l'effet de détour ne dépend pas de la longueur du trajet mais de sa sinuosité relative à la proximité des points d'inflexion.

\section{La contribution des indices de force}

Tous les mouvements biologiques sont le résultat de l'intégration de forces actives et passives (Bernstein 1967). Les forces actives sont les forces musculaires produites par le sujet, au contraire des forces passives, qui sont présentes dans l'environnement, ou bien sur lesquelles le sujet n'a aucune influence, et qui pourtant doivent être prises en compte pour produire le mouvement désiré. Ces forces actives peuvent être aussi modulées par l'augmentation ou la diminution de la «résistance» à un mouvement unidimensionnel produite par le sujet avec ou sans déplacement corporel (force isométrique). L'objectif de cette section est d'examiner si les indices de force jouent un rôle dans la perception kinesthésique des distances, et, le cas échéant, la manière dont elles y contribuent. Le rôle des indices de force dans la perception kinesthésique des distances a peu été étudié. Cependant, 
certaines recherches l'ont indirectement étudié, en variant la condition de l'exploration manuelle.

\subsection{Effet des mouvements actifs versus passifs}

Bien que les mouvements passifs n'interviennent pas souvent dans la vie de tous les jours, il n'en demeure pas moins que l'étude de la perception des mouvements imposés sur une articulation en absence de contraction musculaire peut être considérée comme une première méthode pour étudier la contribution des indices de force dans l'estimation kinesthésique des distances. En effet, dans la condition passive, la décharge corollaire est absente mais les feedbacks kinesthésiques sont générés. Ainsi, la comparaison des performances entre une distance estimée passivement et estimée activement nous donne des résultats intéressants.

Paillard et Brouchon (1968) (cf. aussi Paillard, 1974) ont étudié la précision avec laquelle nous pouvons évaluer la position des membres dans l'espace. Ils ont demandé à des sujets de positionner à un endroit précis leur main droite puis de localiser cet endroit avec la main gauche. Le mouvement des mains est contraint par deux barres verticales dans le plan frontal et sont symétriques par rapport au plan médio-sagittal. Ils se sont intéressés à l'effet de deux types de facteurs susceptibles de coder la position de la main dans l'espace : le positionnement de la main cible (actif versus passif) et la stabilisation de la position de la main cible (maintenue par le sujet ou par l'expérimentateur). Leurs résultats montrent clairement que le système kinesthésique permet de coder plus précisément la position finale d'un bras lorsqu'il est activement déplacé par le sujet que lorsqu'il est déplacé passivement par l'expérimentateur. Par contre, le maintien actif ou passif de la main cible n'a pas d'importance sur les erreurs.

Jones (1974) s'est intéressé au rôle des informations efférentes. D'après lui, les informations issues de la commande motrice sont nécessaires et suffisantes pour le rappel, et 
les informations périphériques auraient un rôle négligeable. Lorsque le sujet effectue activement le mouvement cible, il peut alors se représenter la distance, et par conséquent, l'oubli sera moins important (en rappel immédiat ou après un intervalle de $15 \mathrm{sec}$ ) que lorsque le sujet est passif ou que le mouvement est contraint. Jones conclut que les mouvements présélectionnés sont reproduits grâce à une prise en charge de la copie efférente, à l'opposé des mouvements contraints dont la reproduction dépend essentiellement des informations proprioceptives. Les observations de Stelmach et ses collègues (1975) viennent nuancer les conclusions de Jones (1974). En effet, d'après ces chercheurs, les informations proprioceptives seraient plus importantes que les informations issues de la copie efférente. Le mouvement volontaire faciliterait l'encodage des informations proprioceptives (mais ne serait pas « suffisant et nécessaire »).

La perception/reproduction de mouvements actifs est plus précise que celle de mouvements passifs. Cette différence s'explique avec les mêmes arguments que le contrôle central des efférences. Dans le cas des mouvements exécutés et reproduits activement, le sujet programme son action. Les composantes de ses mouvements sont enregistrées et se révèlent plus précises que celles qui résultent d'un mouvement passif où n'interviennent que les afférences kinesthésiques comme base de comparaison (Kelso, 1977a ; Laszlo et Bairstow, 1985). Toutefois, les différences de précision entre la reproduction de distances parcourues et la localisation finale d'un déplacement du bras, si l'on fait varier le point de départ du bras dans les deux cas, s'expliquent difficilement (Stelmach, Kelso et al., 1975 ; Stelmach, Kelso et al., 1976) : les sujets retrouvent plus facilement le point d'arrêt du mouvement que la distance à parcourir. Il est probable que, dans le cas de la localisation de l'arrêt, le retour à une position de départ pour la reproduction du geste fournisse une afférence que le sujet utilise immédiatement comme indice de "reproduction de distance" en sens inverse, ce qui n'est pas le cas lorsqu'il doit reproduire, à partir d'un point de départ différent, une distance qu'il a 
parcourue une première fois. La combinaison des efférences et des afférences produirait les meilleurs résultats.

\subsection{Le plan de l'espace de travail}

La modification du plan spatial d'exploration manuel (plan horizontal, sagittal, ou frontal) est une seconde méthode pour étudier la contribution des indices de force. Par exemple, la réalisation de la tâche dans le plan frontal détruit la symétrie radiale entre les mouvements dans les différentes directions. Lorsque le plan est frontal, le codage des mouvements requiert un plus haut niveau d'activation musculaire pour s'opposer à la gravité, par rapport aux mouvements dans la direction opposée. Ainsi, l'asymétrie permet de se poser la question du rôle de l'effort moteur dans la perception des propriétés spatiales. En haptique, des recherches ont montré que la perception des longueurs ainsi que l'illusion horizontalvertical $(\mathrm{H}-\mathrm{V})$ sont significativement modifiées par l'orientation du plan (Day et Avery, 1970; Derregowski et Ellis, 1972). Le segment vertical d'une figure en forme de L est surestimé par rapport au même segment dans une orientation horizontale. L'illusion H-V apparait lorsque les mouvements sont réalisés dans le plan horizontal, mais disparaît dans le plan frontal. Les variations dans l'exploration des plans affectent également la perception des autres propriétés

spatiales comme l'orientation (e.g., Gentaz et Hatwell 1995, 1996,1998, 2008) et le parallélisme (e.g., Kappers 2002).

\subsection{Indices d'opposition et de traction}

Wydoodt, Gentaz et al. (2006) ont étudié l'estimation kinesthésique de la longueur d'un segment virtuel. La question centrale était de savoir si les indices de force impliqués dans l'exploration manuelle du stimulus jouent un rôle fondamental dans l'estimation de longueur, et, le cas échéant, la manière dont ils jouent ce rôle. Pour répondre à cette question, deux types d'opposition pendant l'exploration d'un segment étaient proposés dans deux 
premières expériences (oppositions « élastique » ou «visqueuse ») et deux types de traction dans deux autres (tractions «fluide» ou «complète»). Au niveau subjectif, les deux oppositions donnent l'impression de déplacer son doigt dans une "pâte dense ou visqueux » tandis que les deux tractions donnent le sentiment que son doigt est tiré par une corde imaginaire (comme si un élastique étirée se rétractait). Dans ces quatre expériences, Wydoodt et al. (2006) ont utilisé un paradigme classique de psychophysique, fondé sur la comparaison de paires constituées d'un segment normal et d'un segment modifié dynamiquement (Figure 5). Le premier résultat était que les différentes perturbations modifiaient la perception de la longueur d'un segment de $10 \mathrm{~cm}$ dans chaque expérience. Le second résultat était que des biais systématiques opposés étaient observés dans les deux catégories symétriques de perturbation - en opposition ou en traction (voir Figure 6). Ainsi, une sous-estimation de cette longueur était observée avec des perturbations élastiques et visqueuses en opposition, alors qu'une surestimation était obtenue avec des perturbations fluides et complètes de traction. Ces résultats suggèrent que les indices de force influencent l'estimation kinesthésique des longueurs. Pendant l'exploration du stimulus, les indices de forces pourraient jouer un rôle fondamental dans l'estimation des longueurs. Des recherches futures sont requises pour examiner les caractéristiques pertinentes des forces (amplitude, travail, secousse) impliquées dans le mouvement, et pour examiner la manière dont ces indices de forces interagissent avec les indices géométriques dans la perception kinesthésique de l'espace.

(insérer Figure 5) 


\section{Les facteurs cognitifs}

\subsection{L'effet du délai entre la perception et la reproduction}

Dès 1880, Bowditch et Southard montraient que la capacité à déterminer la position de la main dans l'espace avait tendance à se détériorer rapidement sous occlusion visuelle. Des études expérimentales plus récentes (Paillard et Brouchon, 1968 ; Craske et Crawshaw, 1975 ; Wann et Ibrahim, 1992) sont venues conforter cette idée. Certaines d'entre elles reposent sur des observations qui impliquent le port de prismes modifiant la position apparente d'une cible (e.g. Held et Hein, 1958 ; Harris, 1965) : « it seems that kinesthesis manifests an underlying lability when information about the accuracy of its operation is not available. That limb position sense is exceedingly labile is not in doubt ; experiments with prisms (Harris, 1965 ; Craske, 1966) have shown that kinesthesis can be easily modified when vision \& kinesthesis are discordant” (Craskeet Crawshaw, 1975, p 759). Cependant dans une étude récente, Boy, Palluel-Germain et al. (2005) montrent que l'intégration des informations sensorielles nécessaire au codage de la position de la main pouvait également dépendre la nature de la tâche réalisée. Cette expérience a consisté à comparer la production et l'évaluation subjective de mouvements de pointage réalisés dans une situation de contrôle vidéo-assisté. Un dispositif pouvait rendre discordantes les informations visuelles et proprioceptives en biaisant fortement la direction du mouvement perçue sur l'écran (rotation de l'image de 45 degrés). Dans cette situation, alors que les productions motrices des sujets s'adaptent rapidement à ces conditions inhabituelles, on remarque que l'évaluation subjective des aspects spatiaux du mouvement est clairement influencée par l'apparence visuelle du mouvement. En revanche, l'évaluation subjective de la dynamique du mouvement est indépendante de son apparence visuelle et repose sur des informations proprioceptives. La perception que les sujets ont de leur propre mouvement et de la position de leur main dans l'espace de travail semble donc reposer sur des informations qui diffèrent en fonction de la tâche (dynamique vs. spatial). 
La notion selon laquelle la kinesthésie manifeste une labilité prononcée repose également sur le fait que l'estimation de la position d'une main immobile que l'on ne voit pas se détériore notoirement en fonction de l'intervalle de rétention (Wann et Ibrahim, 1992). Cette idée a cependant été remise en question par Desmurget, Vindras et al. (2000). Les auteurs ont demandé à des sujets d'utiliser un joystick avec la main non-dominante afin de pointer un laser en concordance avec la position d'un stylet tenu dans la main dominante, soit immédiatement, soit $10 \mathrm{sec}$ après le positionnement de la main dominante. Dans les deux conditions expérimentales, ni les erreurs constantes systématiques (vecteur reliant la position actuelle de la main et la position indiquée par le sujet), ni les erreurs variables ne se différencient. Ces résultats sont donc en contradiction avec l'hypothèse du déclin de la proprioception à travers le temps. L'interprétation de cette expérience impliquant soit des mouvements de pointage vers des cibles visuelles, soit l'estimation d'une main non visible sous guidage visuel, est néanmoins difficile puisque ces tâches impliquent de complexes transformations visuo-motrices entre les modalités. Par contre, des tâches de reproduction de mouvement cible, de pointage vers des cibles kinesthésiques, de mise en correspondance de la position d'un membre par rapport à un autre, semblent être autant de procédures plus fiables pour étudier l'existence du déclin des informations proprioceptives à travers le temps.

Les données provenant des recherches d'Adams (1967) montrent ainsi que les erreurs augmentent en fonction de la longueur de l'intervalle de rétention (erreur absolue : Posner, 1967 ; Stelmach, 1969 ; Connolly et Jones, 1970 ; Stelmach et Wilson, 1970 ; erreur variable : Adams et Dijkstra, 1966 ; Stelmach et Wilson, 1970 ; Jones, 1974 ; Stelmach, Kelso et al., 1976 ; Kelso, 1977b). Comme nous l'avons vu un peu plus haut, Laabs (1973) observa des erreurs similaires dans la reproduction de la localisation et de la distance à condition que le rappel se fasse immédiatement après la phase de présentation. La supériorité de la précision de la reproduction de la localisation apparaît si un délai d'une vingtaine de secondes sépare la 
phase de présentation de celle du rappel. L'effet du délai interviendrait donc différemment en fonction de l'indice à reproduire.

\subsection{Effet du mode de présentation des distances cibles}

Marteniuk (1973) et Stelmach et al. (1975) rompent avec la tradition d'imposer au sujet la distance cible et demandent alors au sujet de "présélectionner » (Stelmach et al., 1975, Smyth, 1984) cette distance en lui laissant choisir le point d'arrivée du mouvement. Le mouvement était donc déterminé par la commande motrice du sujet et non par la position de la butée. Le sujet devait ensuite retourner au point de départ, puis il devait à nouveau reproduire ce mouvement après un intervalle de rétention. Plusieurs recherches (par exemple Jones 1972 ; Stelmach, Kelso et al. 1975) ont ainsi constaté que la reproduction d'un mouvement présélectionné est plus précise que celle d'un mouvement contraint.

Diverses expériences (Marteniuk, 1973 ; Jones, 1974 ; Stelmach et al., 1975 ; Roy et Diewert, 1975 ; Roy, 1978) ont observé que lorsque les sujets sont autorisés à déterminer la distance du mouvement, alors cette source d'information peut être représentée de manière centrale. Cependant, même lorsque le sujet détermine lui-même le mouvement, le rappel de la localisation finale du mouvement cible se fait de manière plus précise que celui de la distance (Kelso, 1977b). On considère que la présélection du mouvement cible, à travers le traitement central, permet l'utilisation d'un seul indice du mouvement, soit la localisation (Kelso, 1977b), soit la distance (Roy, 1978), indépendamment des afférences associées à la production du mouvement. Stelmach et al. (1976) ont tenté de fournir une explication de la supériorité du rappel, aussi bien pour l'indice de distance que celui de localisation (Roy et Diewert, 1978) ou de l'indice de localisation seul. Il est plus probable que la copie d'efférence et que la congruence avec le feedback kinesthésique dans les mouvements programmés conduisent à de meilleures performances lorsque les mouvements sont présélectionnés, par 
rapport au conflit entre ces facteurs dans les mouvements contraints actifs, et au manque de programme générant une décharge corollaire dans les mouvements passifs.

D'après les travaux de Walsh et de ses collaborateurs, l'indice de distance est une information tout à fait fiable dans la rétention en mémoire à court terme (MCT) motrice. Leurs résultats (Walsh et al., 1979 ; Walsh et Russel, 1979 ; Walsh et Russel, 1980) montrent que le rappel de la distance se fait de manière tout aussi précise que celui de la localisation aussi bien pour les mouvements contraints que pour les mouvements présélectionnés. Walsh et Russel (1979) montrent également que pour des mouvements contraints comme pour des mouvements présélectionnés (Walsh et Russel, 1980), le rappel de la distance est reproduit de manière aussi précise pour des intervalles vides d'une durée de 5 ou de $30 \mathrm{sec}$ (en contradiction avec les résultats de Laabs, 1973 et de Marteniuk et Roy, 1972). Roy et Diewert (1975) observent un meilleur rappel de la distance dans la condition où le sujet présélectionne son mouvement. Aussi, l'influence de l'information de distance sur le rappel de la localisation reste identique après un intervalle de $5 \mathrm{sec}$ ou de $30 \mathrm{sec}$. Enfin, Willberg et Hall (1976) ne trouvent pas de diminution de la précision du rappel de la distance après un délai de $20 \mathrm{sec}$, confirmant ainsi le fait que lorsque le mouvement cible est contraint, alors la distance peut être codée de manière stable.

On peut également observer que dans des situations où le sujet est confronté à la situation de reproduction, il est vraisemblable que la nature du paradigme influence la manière dont le sujet code l'information. Par exemple, si la personne n'a pas d'indice concernant la fin du mouvement référent (mouvement contraint), alors cela peut forcer le sujet à traiter les informations sensorielles concernant la localisation. Ce phénomène pourrait alors le conduire à utiliser une stratégie impliquant un rappel du mouvement effectué grâce à des processus de boucle fermée (système de contrôle employant un feedback, permettant un contrôle des erreurs et une correction, dans le but de maintenir un état désiré). Cependant, 
dans le paradigme où le mouvement cible est un mouvement présélectionné, le sujet peut générer une réponse à l'avance, peut-être même la programmer, et ainsi ignorer les conséquences sensorielles du mouvement simplement en exécutant à nouveau le même programme durant la phase de reproduction. Les mouvements présélectionnés permettent au sujet de planifier tous les paramètres de la réponse à l'avance parce qu'ils sont déterminés par le sujet plutôt que par l'expérimentateur. La planification à l'avance des paramètres du mouvement semble alors jouer en faveur d'une meilleure reproduction du mouvement cible. Parallèlement, le feedback sensoriel apparaît comme étant moins important pour les mouvements présélectionnés que pour les mouvements contraints (Stelmach, 1977 ; en désaccord avec les travaux de Walsh, 1979, 1980, 1981a, b).

\section{Analyse probabiliste des erreurs}

L'examen précédent de la littérature révèle que plusieurs types d'erreurs sont utilisés par les chercheurs et que ces différences dans les usages pourraient expliquer en partie la variabilité des résultats observés. Par exemple, alors que certains (Adams, Marshall et Goetz, 1972a, b ; Jones ,1972) choisissent les erreurs absolues, d'autres (Keele et Ells, 1972 ; Laabs, 1973, 1974) choisissent les erreurs constantes et/ou variables, et d'autres encore (Marteniuk, 1973 ; Stelmach et Kelso 1973 ; Stelmach et al., 1975) préfèrent indiquer ces trois types de variables dépendantes. On peut noter que l'analyse des erreurs finales est également utilisée dans des tâches de pointage dans lesquelles le retour visuel n'est pas perçu pendant l'exécution du mouvement. Lors d'une telle tâche deux types d'erreurs peuvent être dégagés afin de caractériser la distribution des points terminaux. L'erreur constante est alors définie comme la distance entre le point terminal moyen et la position réelle de la cible. L'erreur variable représente la dispersion des points terminaux autour du point terminal moyen. Ces deux types d'erreurs seraient des indices pertinents permettant un accès aux processus de planification impliquées dans une tâche d'atteinte d'objets (e.g., Flanders, Helms-Tillery et 
al., 1992 ; Gordon, Ghilardi et al., 1994 ; Vindras et Viviani, 1998 ; Desmurget, Prablanc et al., 1999).

\subsection{Les différents types de calculs des erreurs}

Nous allons nous intéresser ici plus en détail au calcul des trois types d'erreurs utilisés dans la tâche de positionnement de levier. Rappelons que la tâche consiste à percevoir, puis à reproduire une distance (ou mouvement) cible ( $T$ ), et les réponses peuvent être mesurées selon une unité de mesure (par exemple, en $\mathrm{cm}$ ). Les réponses sont notées $x_{i}$, et $i$ correspond à l'indice pour un essai donné (par exemple, $x_{3}$ est la réponse au troisième essai), sachant que $n$ essais sont réalisés :

L'erreur constante (EC) est calculée comme suit :

$$
\stackrel{n}{n}
$$

On peut obtenir l'erreur absolue par une logique similaire au calcul de l'EC, mais en considérant la valeur absolue de la différence entre la réponse du sujet et la valeur de la distance. L'erreur absolue est obtenue comme suit :

$$
\stackrel{n}{n}
$$

L'erreur variable (EV) est utilisée pour mesurer le degré de consistance des réponses des sujets. Cela concerne la variabilité des réponses du sujet vis-à-vis de la moyenne de ses réponses. On calcule l'EV comme suit :

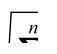

où $\bar{x}$ représente la réponse moyenne des sujets (mesurée avec la même unité que l'ensemble des réponses). 
Cependant, on remarque une disparité à propos des opinions sur la pertinence de ces variables. Selon Laabs (1973), l'EV est la mesure convenable, contrairement à l'opinion défendue par Marteniuk (1973). Cependant, l'EA a été plus souvent au centre des débats (Schutz et Roy, 1973 ; Roy, 1976) car c'est une fonction de l'EV et de l'EC. Lorsque l'EC est nulle ou presque nulle (lorsque le sujet surestime et sous-estime la distance-mouvement cible dans des proportions équivalents), ou lorsque elle est très différente de zéro (les sujets systématiquement surestiment ou sous-estiment la distance de manière constante), alors l'EV et l'EA fournissent la même quantité d'information. Dans le premier cas, l'EC reflète l'étendue des erreurs autour de zéro alors que, dans le second cas, elle indique l'amplitude des erreurs, indépendamment du fait que la distance cible ait été sur- ou sous-estimée. Cependant, si l'EC diffère de zéro parce que le sujet sur- ou sous-estime la distance de manière irrégulière, alors l'EA est une combinaison pertinente de l'EC et de l'EV. Cela a amené Henry (1975) à proposer une « erreur de la variabilité totale » (aussi appelée « erreur quadratique moyenne »). La variabilité totale, notée « E » par Henry (1975) est une mesure composite de l'erreur, et est obtenue en calculant la racine carrée de la somme de $\mathrm{EC}^{2}$ et de $\mathrm{EV}^{2}$, ou sous la forme $E^{2}: E^{2}=E V^{2}+E C^{2}$.

Alternativement, E peut être calculé par la formule suivante :

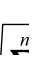

(où $x_{i}, T$ et $n$ correspondent aux paramètres définis préalablement).

Schutz (1977) a été plus loin et a suggéré que l'EA ne devrait pas du tout être utilisée. Cette idée peut être critiquée par le fait que dans certaines situations particulières, il est utile de considérer l'amplitude des erreurs, plutôt que leur signe ou leur variabilité. Roy (1977, 1978) considère l'EA comme une mesure pertinente de la variabilité des réponses, alors que 
l'EC ne serait pas une mesure convenable. D'une manière générale, il est difficile de choisir a priori une variable dépendante.

Lorsque l'on se concentre sur les erreurs pour différentes distances cibles, contrairement à l'EC et l'EA, l'EV reste une mesure qui convient. En effet, ce type d'erreur mesure l'inconsistance dans les estimations des distances par les sujets, quel que soit son étendue. Ceci n'est pas le cas pour l'EA et l'EC. En effet, il est bien plus difficile d'estimer une distance de 100 mètres avec une précision d'un centimètre que d'un mètre, ce qui amène logiquement à l'utilisation d'une erreur relative (ou ratio d'erreur). L'erreur relative est calculée en retirant la mesure réelle de la distance cible à la distance reproduite par le sujet, le tout divisé par la mesure réelle de la distance cible. On peut de plus multiplier le ratio obtenu par cent, pour obtenir un pourcentage d'erreur. Par exemple, dans une tâche dans laquelle le sujet doit estimer et reproduire des distances de 5 et $20 \mathrm{~cm}$, les erreurs mesurées n'ont pas la même signification et peuvent amener à des conclusions opposées. Supposons que pour chacune de ces distances, le sujet les surestime de 2 et $4 \mathrm{~cm}$, respectivement. Au premier abord, on peut remarquer que les erreurs sont deux fois plus importantes pour la reproduction d'une grande distance par rapport à une distance courte. Si l'on considère ces erreurs telles quelles, on peut conclure que la perception/reproduction d'un mouvement d'une distance de 5 cm est bien plus précise que celle d'une distance de $20 \mathrm{~cm}$. En revanche, si l'on considère les erreurs relatives, alors elles sont de 0,4 (40\%) dans le premier cas et seulement de 0,2 (20\%) dans le second cas. En d'autres termes, les erreurs relatives montrent que la reproduction d'une distance de $5 \mathrm{~cm}$ est deux fois plus précise que celle d'une distance de $20 \mathrm{~cm}$. Finalement, nous constatons avec surprise que l'erreur relative a très rarement été utilisée : dans l'ensemble des articles considérés publiés entre 1964 et 1992, seuls Adams, Goetz et al. (1972a, 1972b) l'ont utilisée. 


\subsection{Apport de l'analyse probabiliste}

Nous proposons un point de vue probabiliste sur la question ci après : comment comparer théoriquement ces différentes mesures d'erreurs? L'une d'entre elles est-elle formellement plus adéquate, pour une expérience de mémorisation et de reproduction de distances unidimensionnelles ? Cette question est-elle bien posée ? Nous pensons que non : en réalité chaque mesure d'erreur fait référence à des hypothèses implicites différentes qui peuvent être satisfaites, ou non, par le système considéré. En effet, l'EC et l'EV font l'hypothèse d'une distribution gaussienne (normale) des réponses des sujets, alors que l'EA fait l'hypothèse d'une distribution laplacienne de ces réponses (Tarentola, 2006). Nous souhaitons maintenant clarifier et démontrer ce résultat. En effet, l'EC est une mesure directe de la moyenne de la distribution de probabilités gaussienne, sur laquelle on fait l'hypothèse que les sujets tirent aléatoirement leurs réponses. Cela peut être vu en réécrivant la définition de l'EC:

$$
\begin{aligned}
C E & =\frac{\sum_{i=1}^{n}\left(x_{i}-T\right)}{n} \\
& =\frac{\sum_{i=1}^{n} x_{i}-\sum_{i=1}^{n} T}{n} \\
& =\frac{\sum_{i=1}^{n} x_{i}}{n}-\frac{n T}{n} \\
C E & =\bar{x}-T
\end{aligned}
$$

où $\bar{x}$ est l'estimation de la moyenne de l'ensemble des réponses $x_{i}$. Ainsi, l'EC est en réalité cette moyenne, translatée de $-T$, ce qui est juste un changement d'origine.

L'EV est tout simplement la définition de l'écart-type des réponses $x_{i}$. Notons cependant que, lorsque l'EC et l'EV sont estimées sur le même ensemble de réponses $x_{i}$, l'EV définie strictement par

$$
V E=\sqrt{\frac{\sum_{i=1}^{n}\left(x_{i}-\bar{x}\right)^{2}}{n}}
$$


est un estimateur biaisé de l'écart-type. Il serait plus approprié d'utiliser la version non biaisée de cet estimateur :

$$
V E^{\prime}=\sqrt{\frac{\sum_{i=1}^{n}\left(x_{i}-\bar{x}\right)^{2}}{n-1}}
$$

Cependant, notons que le ratio des deux mesures est $n-1 / n$; lorsque $n$ est grand, les deux indices tendent asymptotiquement l'un vers l'autre.

Calculer l'EC et l'EV à partir d'un ensemble de réponses $x_{i}$ revient à calculer la distribution de probabilité gaussienne de maximum de vraisemblance. En d'autres termes, ce calcul fait l'hypothèse que les réponses des sujets ont été tirées aléatoirement sur une distribution de probabilités gaussienne, de paramètres $\mu, \sigma$ inconnus. On cherche alors les valeurs $\bar{\mu}, \bar{\sigma}$ qui maximisent la probabilité d'obtenir les $N$ données $x_{i}$ : cette probabilité s'appelle la vraisemblance du jeu de données. Nous avons donc montré, plus haut, que $\bar{\mu}=$ $E C+T$ et que $\bar{\sigma}=E V$.

La troisième mesure d'erreur, l'EA, suit exactement le même raisonnement : c'est un paramètre estimé d'une distribution de probabilités de maximum de vraisemblance (Figure 6). La différence est que la classe de distributions ici considérée est l'ensemble des distributions de probabilités de Laplace (Karst et Polowy, 1963).

(insérer Figure 6)

Une distribution de probabilités de Laplace est uni-modale (elle a un pic unique) et symétrique autour de ce pic, comme une distribution gaussienne. La fonction de densité d'une distribution de probabilités de Laplace est définie par :

$$
L_{a, b}(x)=P(x \mid a, b)=\frac{1}{2 b} e^{-\frac{|x-a|}{b}}
$$

où $a$ est le pic de la distribution, et $b>0$ est sa dispersion (voir Figure 7). 
Étant donné un ensemble $x_{i}$ de réponses d'un sujet, la distribution de probabilité de Laplace de maximum de vraisemblance a pour paramètres $\bar{a}, \bar{b}$ (Karst et Polowy, 1963 ; Chen, 2002) :

$$
\begin{aligned}
& \bar{a}=\operatorname{median}\left(x_{i}\right) \\
& \bar{b}=\frac{\sum_{i=1}^{n}\left|x_{i}-\bar{a}\right|}{n}
\end{aligned}
$$

On rappelle que la médiane d'un ensemble d'échantillons $x_{i}$ est la valeur telle qu'il y a autant d'échantillons plus petits qu'il n'y a d'échantillons plus grands que cette valeur. En d'autres termes, c'est la valeur qui coupe l'ensemble d'échantillons en deux sous-ensembles de même taille.

Lorsque le pic est supposé connu et à une valeur $\bar{a}$, le paramètre de dispersion de maximum de vraisemblance $\bar{b}$ est calculé par (Chen, 2002) :

$$
\bar{b}=\frac{\sum_{i=1}^{n}\left|x_{i}-\bar{a}\right|}{n}
$$

Ce dernier calcul montre que calculer la mesure d'erreur absolue EA revient à calculer la distribution de probabilités de Laplace de maximum de vraisemblance, sous l'hypothèse que le pic est connu, et à la valeur $\bar{a}=T$, avec $T$ la mesure du mouvement cible que les sujets devaient reproduire. Ceci conclut la démonstration du fait que l'EC et l'EV sont les paramètres décrivant respectivement la moyenne et l'écart-type d'une distribution gaussienne, et que l'EA décrit la dispersion d'une distribution de Laplace (sous l'hypothèse que son pic est en T).

En conclusion, dans une expérience de mémorisation et de reproduction de mouvements unidimensionnels, quelle mesure d'erreur devrait être préférée ? Les définitions ci-dessus montrent que cette question est mal posée. Calculer l'EC revient à résumer la répartition des points expérimentaux par le calcul de la moyenne d'une gaussienne, calculer l'EV revient résumer la répartition de ces points par leur écart-type, et, finalement, calculer l'EA revient à résumer la répartition des points expérimentaux par la dispersion d'une 
distribution de Laplace, sous l'hypothèse qu'elle est centrée sur $T$. Notons que nous considérons bien ici uniquement les distributions de probabilité résumant les répartitions des points expérimentaux, et non les répartitions des mesures d'erreurs (sur plusieurs sujets, par exemple).

Chacune de ces mesures correspond donc à un ensemble différent d'hypothèses sur la façon dont on résume les données expérimentales. Discuter de la pertinence des mesures ne prend de sens qu'au vu des modèles sous-jacents. Ainsi, la question peut être reformulée en première approche en une question qui concerne les distributions de réponses des sujets : ces réponses sont-elles distribuées selon une loi gaussienne ou une loi de Laplace?

Il y a bien sûr un argument théorique très fort en faveur des modèles basés sur des distributions de probabilités gaussiennes : le Théorème Central Limite. En termes simples, ce théorème énonce que des données approximent des distributions gaussiennes lorsque ces données résultent d'un effet principal, et d'une somme d'un grand nombre d'effets secondaires, qui sont de plus petite magnitude que l'effet principal, et qui ne sont pas corrélés. Dans le paradigme de positionnement de levier, il se pourrait que les réponses des sujets soient principalement dues à leur mémoire d'une distance (ou position) à reproduire, mais qu'elles soient également perturbées par plusieurs effets de plus petite échelle, comme l'imprécision de la mémoire, le biais de l'appareillage expérimental, ou encore les erreurs de mesures. Dans ce cas, on pourrait alors faire l'hypothèse que les réponses des sujets sont distribuées selon une distribution gaussienne.

Cependant, les hypothèses ci-dessus peuvent être ou ne pas être satisfaites. Il se pourrait que certains des effets de petite échelle soient corrélés, ou bien que leur magnitude ne soit pas si petite comparée à la magnitude de la distance $T$ à reproduire. Dans ces cas, la distribution de probabilités gaussienne n'est plus forcément la meilleure hypothèse. Par exemple, il se pourrait que la mémoire à court terme donne en sortie une mesure $T_{1}$ à 
reproduire et que, ayant cette valeur en consigne, le système mécanique de déplacement du bras arrive à une mesure $T$. Cette mesure, qui est observée expérimentalement, pourrait incorporer un biais systématique par rapport à $T_{1}$. Dans ce cas, les réponses observées des sujets devraient être modélisées par le résultat de deux effets principaux corrélés. Un biais systématique pourrait être représenté par une distribution de probabilités asymétrique : dans ce cas, ni la distribution gaussienne ni la distribution de Laplace ne conviendraient.

Dans d'autres cas, lorsque les points expérimentaux sont bien répartis symétriquement, mais sont en réalité plus dispersés que ne l'aurait prédit une analyse fondée sur une distribution de probabilités gaussienne, une distribution de probabilité de Laplace peut être plus adéquate. En effet, les distributions de probabilités de Laplace décroissent plus lentement que les gaussiennes lorsqu'on s'écarte du pic : on dit que ce sont des distributions à longue ou large queue (« heavy-tailed» en anglais). En tant que telles, elles ont été appliquées avec succès à la modélisation de signaux de parole ou d'images (Eltoft, Kim et al., 2006).

De plus, il est peut-être très difficile de décider expérimentalement si un ensemble de points suit une distribution gaussienne, ou de Laplace, ou encore d'un autre type. Ceci est d'autant plus vrai que l'ensemble de points est petit, ce qui est couramment le cas dans le cas des expériences de psychologie expérimentale (par rapport à certains domaines en sciences physiques, par exemple). Considérons par exemple l'ensemble de données montré Figure 7, et les distributions gaussiennes et de Laplace de maximum de vraisemblance qui en résultent. Il serait difficile de trancher et de sélectionner l'un de ces deux modèles avec confiance.

(insérer Figure 7)

Une approche bien plus méthodique consisterait à reprendre les données existantes, et leur appliquer des tests statistiques pour déterminer si elles sont distribuées selon des 
distributions gaussiennes, de Laplace (Chen, 2002), ou selon d'autres distributions, possiblement asymétriques, voire même non uni-modales. Cela ne suffirait pas en soi, car il serait aussi intéressant de déterminer si tous ces hypothèses représentent correctement les données, ou bien si l'une d'entre elle est, dans tous les cas expérimentaux, bien meilleure que les autres. Cela pourrait être fait par une comparaison systématique des vraisemblances de ces hypothèses.

Plus généralement, cette approche montre qu'il n'y a pas de mesure d'erreur, que ce soit l'EA, l'EV, l'EC ou bien encore une autre, qui soit formellement meilleure que les autres. Elles sont liées à des modèles implicites qui font leur lot d'hypothèses, et qui ne sont pas facilement discriminables expérimentalement. Une comparaison méthodique de ces hypothèses devrait être réalisée par une étude de comparaison et de sélection de modèles.

\section{Conclusion générale}

Bien que de nombreuses différences existent entre les études sur la reproduction des distances, on peut en tirer quelques résultats consistants sur la contribution des indices géométrique : 1) les distances courtes sont généralement surestimées et les distances longues sont généralement sous-estimées, et 2) l'interférence entre distances et positions suit un pattern stéréotypé. Le mouvement de reproduction vers la position finale est hypométrique lorsque le point de départ varie de manière à ce que la distance à parcourir est plus longue que le mouvement cible, et il est hypermétrique lorsque la distance est plus courte. À l'inverse, la distance cible est sous-estimée lorsque le point de départ est rapproché du point final, et elle est surestimée lorsque le point de départ en est éloigné. Ces résultats soulignent l'importance des indices géométriques dans la perception kinesthésique des distances ; cependant, les indices de forces et les facteurs cognitifs restent des variables importantes. Le rôle des indices de force dans la perception kinesthésique des distances a été étudié par des méthodes expérimentales variées : soit en changeant le rôle du sujet, le plan de l'espace de travail, ou les 
indices de traction et d'opposition. Les études présentées dans cet article montrent que ces facteurs peuvent intervenir dans la perception de l'étendue du mouvement. En effet, la distance et la position des mouvements libres et actifs sont reproduites avec plus de précision pour des mouvements avec butée, ou pour des mouvements passifs. Le plan de travail semble également jouer un rôle significatif dans l'illusion vertical-horizontal, et dans la perception d'autres propriétés spatiales comme l'orientation et le parallélisme. De plus, les indices de traction et d'opposition pourraient être extrêmement labiles, bien que des expériences récentes aient montré que la perception de propriétés spatiales était significativement modifiée par ces indices. Finalement, il a été montré que le délai et le mode de présentation du mouvementcible affectaient les performances. En effet, diverses expériences ont montré la labilité de la kinesthésie et l'importance de la copie efférente dans les conditions en mouvements présélectionnés. L'ensemble des résultats confirme notre hypothèse selon laquelle les humains ne se représentent pas mentalement les distances perçues via un mouvement de la main dans un espace euclidien.

Par ailleurs, les recherches présentées dans cette revue soulignent les relations étroites entre les propriétés encodées pendant la production de mouvement. Plus spécifiquement, les propriétés géométriques, dynamiques, et les facteurs cognitifs (par exemple, le délai entre la présentation et la reproduction $\mathrm{du}$ mouvement cible) semblent être étroitement interdépendants. Ainsi, pour étudier la perception kinesthésique des propriétés spatiales, il est nécessaire de prendre en compte à la fois les indices géométriques, les indices de force, et les facteurs cognitifs. Il est très probable que cette approche puisse être fructueuse pour aborder la perception haptique des orientations car plusieurs études révèlent que les indices géométriques et de force (e. g. Gentaz \& Hatwell, 2006) ainsi que les facteurs cognitifs (e.g., Gentaz \& Hatwell, 1999) influencent les performances dans les reproductions des orientations d'une baguette avec une main. Ce constat impose de sérieuses contraintes pour obtenir un 
modèle mathématique du système complet mis en jeu une tâche d'estimation kinesthésique des propriétés spatiales. En effet, ce modèle devrait intégrer toutes les parties du système physique de contrôle, en allant de la forme et la dynamique du bras, jusqu'aux caractéristiques de labilité du système mémoriel chargé de la rétention des indices. Il devrait également être largement couplé aux autres modalités sensorielles que la kinesthésie, en intégrant notamment la vision, car il semble de plus en plus vraisemblable que la multi-modalité soit plus la norme que l'exception dans le traitement sensori-moteur, comme suggéré par les récentes recherches en perception multi-modale. Enfin, un modèle complet du bras, pour être satisfaisant, devrait être général quant à la tâche traitée : laissé en mouvement libre dans l'espace 3D, il devrait rendre compte des observations sur les tâches expérimentales de pointage ; contraint aux mouvements à deux dimensions sur le plan d'une table, il devrait rendre compte des capacités d'écriture ; enfin, contraint aux mouvements mono-dimensionnels le long d'un rail, il devrait rendre compte des observations sur le paradigme de positionnement de levier. Ceci bien sûr, si l'on suppose des caractéristiques communes, un système de contrôle commun à toutes les tâches réalisées par le bras, ce qui reste une hypothèse à confronter à l'expérience. Les développements récents en modélisation probabiliste des systèmes sensori-moteurs offrent une piste prometteuse, notamment grâce à la capacité des modèles probabilistes à utiliser un formalisme mathématique cohérent pour la fusion des informations sensorielles diverses (aussi bien cinématiques que dynamiques) et des informations motrices (comme la production des forces, et la prédiction de leurs effets). 


\section{Références bibliographiques}

Adams, J. A. \& S. Dijkstra (1966). "Short-term memory for motor responses." Journal of Experimental Psychology $71: 314-318$.

Adams, J. A., E. T. Goetz, et al. (1972a). "Response feedback \& motor learning." Journal of Experimental Psychology 92 : 391-397.

Adams, J. A., E. T. Goetz, et al. (1972b). "Response feedback \& short-term motor retention." Journal of Experimental Psychology 92 : 92-95.

Bernstein, N. (1967). The coordination \& regulation of movements. Oxford, Pergamon.

Blumenfeld, W. (1937). "The relationship between the optical \& haptic construction of space." Acta Psychologica 2 : 133-157.

Boy, F., R. Palluel-Germain, et al. (2005). "Dissociation between "where" \& "how" judgments of one's own motor performance in a video-controlled reaching task." Neuroscience letters 386 : 52-57.

Chen, C. (2002). "Tests for the goodness-of-fit of the Laplace distribution." Communications in Statistics - Simulation \& Computation $31: 159-174$.

Clark, F. J. \& K. W. Horch (1986). Kinaesthesia. Handbook of perception \& human performance. K. R. Boff, L. Kaufman \& J. P. Thomas. New York, John Wiley \& Sons : 13.1-13.62.

Colley, A. \& M. Colley (1981). "Reproduction of end location \& distance of movement in early \& later blind subjects." Journal of Motor Behavior 13 : 102-109.

Connolly, K. \& B. Jones (1970). "A developmental study of afferent-reafferent integration." Bristish Journal of Psychology 61 : 259-266.

Craske, B. \& M. Crawshaw (1975). "Shifts in kinesthesis through time \& after active \& passive movement." Perceptual \& Motor Skills 40 : 755-761.

Cuijpers, R. H., A. M. Kappers, et al. (2000). "Large systematic deviations in visual parallelism." Perception 29 : 1467-1482. 
Cuijpers, R. H., A. M. Kappers, et al. (2002). "Visual perception of collinearity." Perception \& Psychophysics 64 : 1467-1482.

Day, R. H. \& G. C. Avery (1970). "Absence of the horizontal-vertical illusion in haptic space." Journal of Experimental Psychology 83 : 172-173.

Derregowski, J. \& H. D. Ellis (1972). "Effect of stimulus orientation upon haptic perception of the horizontal-vertical illusion." Journal of Experimental Psychology 95 : 14-19.

Desmurget, M., C. Prablanc, et al. (1999). "Are reaching movements planned to be straight \& invariant in the extrinsic space? Kinematic comparison between compliant \& unconstrainted motions." Quartely Journal of Experimental Psychology 52 : 981-1020.

Desmurget, M., P. Vindras, et al. (2000). "Proprioception does not quickly drift during visual occlusion." Experimental Brain Research 134 : 363-377.

Diewert, G. L. (1975). "Retention \& coding in motor short-term memory : a comparison of storage codes for distance \& location information." Journal of Motor Behavior 7 : 183 190.

Eltoft, T., T. Kim, et al. (2006). "On the multivariate Laplace distribution." IEEE Signal Processing Letters 13 : 300-303.

Faineteau, H., E. Gentaz, et al. (2003). "The kinaesthetic estimation of Euclidean distances in blindfolded adults : A study on the detour path effect." Experimental Brain Research 153 : 166-172.

Faineteau, H., E. Gentaz, et al. (2005). "Factors affecting the size of the detour effect in the kinaesthetic perception of Euclidian distance." Experimental Brain Research 163 : 503-514.

Faineteau, H., R. Palluel-Germain, et al. (2007). "Effets des points d'inflexion sur les estimations des distances euclidiennes dans une tâche manuelle d'intégration des trajets." Année Psychologique 1 : 3-14.

Flanders, M., S. I. Helms-Tillery, et al. (1992). "Early stages in a sensori-motor transformation." Behavioral \& Brain Sciences 15 : 309-362.

Gandevia, S. C. (1996). Kinesthesia : Roles for afferent signals \& motor commands. Handbook of physiology : a critical, comprehensive presentation of physiological 
knowledge \& concepts. L. B. Rowell \& J. T. Shepherd. New York, Oxford University Press. Section 12 : Exercise : regulation \& integration of multiple systems, 128-172.

Gandevia, S. C. \& D. Burke (1992). "Does the nervous system depend on kinesthetic information to control natural limb movements?" Behavioral \& Brain Sciences 15 : 614-632.

Gentaz, E. \& Gaunet, F. (2006). L'inférence haptique d'une localisation spatiale chez les adultes et les enfants: étude de l'effet du trajet et de l'effet du délai dans une tâche de complétion de triangle. Année Psychologique, 2, 167-190.

Gaunet, F. \& E. Gentaz (sous presse). "Effet de l'expérience visuelle sur l'inférence de la localisation spatiale." Année Psychologique.

Gentaz, E. \& Y. Hatwell (1995). "The haptic "oblique effect" in children's \& adults' perception of orientation." Perception 24 : 631-646.

Gentaz, E. \& Y. Hatwell (1996). "Role of gravitational cues in the haptic perception of orientation." Perception \& Psychophysics 58 : 1278-1292.

Gentaz, E. \& Y. Hatwell (1998). "The haptic oblique effect in the perception of rod orientation by blind adults." Perception \& Psychophysics 60 : 157-167.

Gentaz, E., \& Hatwell, Y. (1999). Role of memorisation conditions in the haptic processing of orientations and the "oblique effect". British Journal of Psychology, 90, 373-388.

Gentaz , E. \& Y. Hatwell (2008). Haptic perceptual illusions. Human haptic perception. M. Grunwald. Berlin, Birkhäuser (pp. 223-233).

Gentaz, E., Y. Hatwell, et al. (2006). Percevoir l'espace avec les mains. Traité de la Réalité Virtuelle 3, volume 1. P. Fuchs. Paris, Ecole des Mines de Paris : (pp.265-289).

Gordon, J., M. F. Ghilardi, et al. (1994). "Accuracy of planar reaching movements. I. Independence of direction \& extent variability." Experimental Brain Research 99 : 97 111.

Gundry, J. (1975). "The use of location \& distance in reproducing diffrent amplitudes of movements." Journal of Motor Behavior 7 : 91-100.

Hagman, J. D. \& E. W. Francis (1975). "The instructional variable \& kinesthetic cue recall." Journal of Motor Behavior 7 : 141-146. 
Hall, C. \& R. B. Wilberg (1977). "Distance reproduction velocity \& the range effect." Journal of Human Movement Studies 3 : 60-65.

Harris, C. S. (1965). "Perceptual adaptation to inverted, reversed, \& displaced vision." Psychological Review 72 : 419-444.

Held, R. \& A. Hein (1958). "Adaptation of disarranged hand-eye coordination contingent upon re-afferent stimulation." Perceptual \& Motor Skills 8 : 87-90.

Henry, F. M. (1975). "Absolute error versus "E" in target accuracy." Journal of Motor Behavior 7 : 227-228.

Hermelin, N. \& B. O'Connor (1975). "Location \& distance estimates by blind \& sighted children." Quarterly Journal of experimental Psychology 27 : 295-301.

Imanaka, K. (1989). "Effect of starting position on reproduction of movement : Further evidence of interference between location \& distance information." Perceptual \& Motor Skills 68 : 423-434.

Imanaka, K. \& B. Abernethy (1992). "Cognitive strategies \& short-term memory for movement distance \& location." Quartely Journal of Experimental Psychology 45A : 669-700.

Imanaka, K. \& B. Abernethy (1992a). "Cognitive strategies \& short-term memory for movement distance \& location." The Quarterly Journal of Experimental Psychology 45 : 669-700.

Imanaka, K. \& B. Abernethy (1992b). "Interference Between Location \& Distance Information in Motor Short-Term Memory : The Respective Roles of Direct Kinesthetic Signals \& Abstract Codes." Journal of Motor Behavior 24 : 274-280.

Imanaka, K. \& B. Abernethy (2000). Distance-location interference in movement reproduction : An interaction between conscious \& unconscious processing? Beyond dissocation : Interaction between dissociated implicit \& explicit processing. Y. Rossetti \& A. Revonsuo. Amsterdam : John Benjamins : pp. 41-71.

Imanaka, K., Y. Nishihira, et al. (1996). "Interference between location \& distance information in the reproduction of arm positioning : Early implications \& new directions." Advances in Exercise \& Sport Physiology 2 : 1-12. 
Johnson, R. W. \& R. W. Simmons (1980). "Extent of movement, terminal position, \& practice as determinants of the range effect." Perceptual \& Motor Skills 50 : 10191022.

Jones, B. (1972). "Outflow \& inflow in movement duplication." Perception \& Psychophysics 12 : $95-96$.

Jones, B. (1974). "Role of central monitoring of efference in short-term memory for movements." Journal of Experimental Psychology 102 : 37-43.

Kandel, E. \& J. Schwartz (1991). Principles of Neural Science. New York, Elsevier.

Kappers, A. (2002). "Haptic perception of parallelity in the midsagittal plane." Acta Psychologica $109: 25-40$.

Kappers, A. M. (1999). "Large systematic deviations in the haptic perception of parallelity." Perception 28 : 1001-1012.

Kappers, A. M. \& J. J. Koenderink (1999). "Haptic perception of spatial relations." Perception 28 : 781-795.

Karst, O. \& H. Polowy (1963). "Sampling properties of the median of a Laplace distribution." American Mathematical Monthly 70 : 628-636.

Keele, S. W. (1968). "Movement control in skilled motor performance." Psychological Bulletin 6 : 387-403.

Keele, S. W. \& J. G. Elis (1972). "Memory characteristics of kinesthetic information." Journal of Motor Behaviour 4 : 127-134.

Keele, S. W. \& J. G. Ells (1972). "Memory characteristics of kinesthetic information." Journal of Motor Behavior 4 : 387-403.

Kelso, J. A. (1977a). "Motor control mechanisms underlying human movement reproduction." Journal of Experimental Psychology : Human Perception \& Performances 3 : 529-543.

Kelso, J. A. (1977b). "Planning \& efferent components in the coding of movement." Journal of Motor Behavior 4 : 124-134.

Kerr, R. M. (1978). "The effect of invalid task parameters on short-term motor memory." Journal of Motor Behavior 10 : 261-273.

Laabs, G. J. (1973). "Retention characteristics of different reproduction cues in motor shortterm memory." Journal of Experimental Psychology 100 : 168-177. 
Laabs, G. J. (1974). "The effect of interpolated motor activity on short-term retention of movement distance \& end-location." Journal of Motor Behavior 6 : 279-288.

Laabs, G. J. \& R. W. Simmons (1981). Motor memory. Human Skills. D. H. Holding. Chichester, Engl \& : Wiley.

Laszlo, J. I. \& P. J. Bairstow (1985). Perceptual Motor Behavior. Developmental Assessment \& Therapy. London, Holt Ltd.

Lederman, S. J., R. L. Klatzky, et al. (1985). "Spatial \& movement-based heuristics for encoding pattern information through touch." Journal of Experimental Psychology: General $114: 33-49$.

Lederman, S. J., R. L. Klatzky, et al. (1987). "Exploring environments by h \& or foot : Timebased heuristics for encoding distance in movement space." Journal of Experimental Psychology : Memory \& Language 16 : 606-614.

Marteniuk, R. G. (1973). "Retention characteristics of motor short-term memory cues : A reply to Laabs [Notes \& Comments]." Journal of Motor Behavior 7 : 151-152.

Marteniuk, R. G. (1976). Cognitive information processes in motor short-term memory \& movement production. Motor Control : Issues \& Trends. G. E. Stelmach. Academic Press, New York.

Marteniuk, R. G. \& E. A. Roy (1972). "The codabilty of kinesthetic location \& distance information." Acta Psychologica 36 : 471-479.

Marteniuk, R. G., K. W. Shields, et al. (1972). "Amplitude, position, timing \& velocity as cues in reproduction of movement." Perceptual \& Motor Skills 35 : 51-58.

Matthews, P. B. C. (1988). "Proprioceptors \& their contribution to somatosensory mapping : complex messages require complex processing." Canadian Journal of Physiology \& Pharmacology $66: 430-438$.

McCloskey, D. I. (1977). "Corollary motor discharges \& kinaesthesia." Brain Research 63 : 119-131.

Millar, S. (1994). Understanding \& representing space. Theory \& evidence from studies with blind \& sighted children. Oxford, Clarendon Press.

Paillard, J. (1974). Le traitement des informations spatiales. De l'espace corporel à l'espace écologique. J. Bresson, P.-H. Chombart de Lauwe, M. Cullenet al. Paris, P.U.F. : 7-54. 
Paillard, J. \& M. Brouchon (1968). Active \& passive movement in the calibration of position sense. The neuropsychology of spatially oriented behavior. S. J. Freedman. Homewood, Ill. : 35-55.

Pepper, R. L. \& L. M. Herman (1970). "Decay \& interference effects in the short-term retention of a discrete motor act." Journal of Experimental Psychology 83 : 1-18.

Posner, M. I. (1967). "Characterictics of visual \& kinesthetic memory codes." Journal of Experimental Psychology 75 : 103-107.

Proske, U. (2005). "What is the role of muscle receptors in proprioception." Muscle Nerve 31 : 780-787.

Proske, U., A. K. Wise, et al. (2000). "The role of muscle receptors in the detection of movements." Progress in Neurobiology 60 : 85-96.

Robles-de-la-Torres, G. \& V. Hayward (2001). " Force can overcome object geometry in the perception of shape through active touch,." Nature 412 : 445-448.

Roll, J.-P. (1994). Sensibilités cutanées et musculaires. Traité de Psychologie Expérimentale 1. M. Richelle, J. Requin \& M. Robert. Paris, P.U.F : pp. 483-542.

Rosembaum, D. A., L. D. Loukolopoulos, et al. (1995). "Planning reaches by evaluating stored postures." Psychological Review 102 : 28-67.

Roy, E. A. (1976). "Measuring change in motor memory." Journal of Motor Behavior 8 : 283288.

Roy, E. A. (1977). "Spatial cues in memory for movement." Journal of Motor Behavior 9 : 151-156.

Roy, E. A. (1978). "Role of preselection in memory for movement extent." Journal of Experimental Psychology : Human Learning \& Memory 4 : 397-405.

Russel, D. G. (1976). Spatial location cues \& movement production. Motor Control : Issues \& Trends. G. E. Stelmach. New-York, Academic Press : pp. 67-83.

Schutz, R. W. (1977). Absolute, constant, \& variable error : Problems \& solutions. Proceedings of the Colorado Measurement Symposium. D. Mood. Boulder, CO : University : pp. 82-100.

Smyth, M. M. (1984). Memory for movements. The Psychology of Human Movement. M. M. Smyth \& A. Wing. Academic Press, New York. 
Stelmach, G. \& M. Wilson (1970). "Kinesthetic retention, movement extent, \& information processing." Journal of Experimental Psychology 85 : 425-430.

Stelmach, G. E. (1969). "Prior positioning responses as a factor in short-term memory of a simple motor task." Journal of Experimental Psychology 81 : 523-526.

Stelmach, G. E. (1973). "Feedback - a determiner of forgetting in short term motor memory." Acta Psychologica 37 : 333-339.

Stelmach, G. E. \& S. L. Bassin (1971). "The role of overt motor rehearsal in kinesthetic recall." Acta Psychologica 35 : 56-63.

Stelmach, G. E., J. A. Kelso, et al. (1976). "Preselection \& response biasing in short-term memory." Memory \& Cognition 4 : 62-66.

Stelmach, G. E., J. A. Kelso, et al. (1975). "Preselection in short-term motor memory." Journal of Experimental Psychology : Human Learning \& Memory 1 : 745-755.

Tarentola, A. (2006). "Popper, bayes \& the inverse problem." Nature Physics 2 : 492-494.

Toole, T., R. W. Christian, et al. (1982). "Preselected movement accuracy as a function of movement time, distance \& velocity." Journal of Human Movement Studies 8 : 93 102.

Vindras, P. \& P. Viviani (1998). "Frames of reference \& control parameters in visuo-manual pointing." Journal of Experimental Psychology : Human Perception \& Performance 24 : 569-591.

Walsh, M. F. (1981a). "Memory for preselected \& constrained short movements." Research Quarterly for Exercise \& Sport $52: 368-379$.

Walsh, M. F. \& D. G. Russel (1979). "Memory for movement location \& distance : starting position \& retention interval effect." Journal of Human Movement Studies 5 : 68-76.

Walsh, M. F. \& D. G. Russel (1980). "Memory for preselected slow movements : evidence for integration of location \& distance." Journal of Human Movement Studies 6 : 96105.

Walsh, M. F., D. G. Russel, et al. (1981b). "Interference effects in recalling movements." British Journal of Psychology 72 : 287-298. 
Walsh, M. F., D. G. Russel, et al. (1979). "Memory for constrained \& preselected movement location \& distance : effect of starting position \& length." Journal of Motor Behavior $11: 201-214$.

Wann, J. P. \& S. F. Ibrahim (1992). "Does limb proprioception drift?" Experimental Brain Research 91 : 162-166.

Wilberg, R. B. \& Y. Girouard (1976). "Distance perception affected by retention of the stimuli in short-term memory." Canadian Journal of Psychology 30 : 63-71.

Williams, H. L., W. S. Beaver, et al. (1969). "Digital \& kinesthetic memory with interpolated information processing." Journal of Experimental Psychology $80: 530-536$.

Wrisberg, C. A., D. G. Millslagle, et al. (1987). "Reproducing the endpoint of a positioning movement : a further test of the influence of start location." Journal of Human Movement Studies 13 : 391-398.

Wrisberg, C. A. \& T. P. Winter (1985). "Reproducing the end location of a postioning movement : the long \& short of it." Journal of Motor Behavior $17: 242-254$.

Wydoodt, P., E. Gentaz, et al. (2006). "Role of force cues in the haptic estimations of a virtual length." Experimental Brain Research 171 : 481-489. 


\section{Légende des figures}

Figure 1 : Effet d'interférence entre la distance et la localisation sur les erreurs constantes (Imanaka \& Abernethy, 2000)

Figure 1 : Effect of the interference between distance and location on constant errors (Imanaka \& Abernethy, 2000)

Figure 2 : Longueurs des trajets d'exploration durant la phase d'encodage dans la condition où l'encodage et la réponse se trouvent au niveau de l'axe médian du sujet (Faineteau, Gentaz \& Viviani 2003): 1) Petit espace $\mathrm{S} 1: \mathrm{A}=1 \times 7.5 \mathrm{~cm}, \mathrm{~B}=2 \times 7.5 \mathrm{~cm}$ et $\mathrm{C}=3 \times 7.5 \mathrm{~cm}$;2) Grand espace $\mathrm{S} 2=\mathrm{D}=1 \times 22.5 \mathrm{~cm}, \mathrm{E}=2 \times 22.5 \mathrm{~cm}$ et $\mathrm{F}=3 \times 22.5 \mathrm{~cm}$. L'estimation de la distance absolue est donnée toujours dans le même segment.

Figure 2 : Outlay of the workplane. Each scale (S1: $\{A, B, C\}$ and S2: $\{D, E, F\})$ included one straight, and two variable-curvature paths (Faineteau, Gentaz \& Viviani 2003). The straight paths (A and D) were $7.5 \mathrm{~cm}$ and $22.5 \mathrm{~cm}$ long, respectively, and were parallel to the sagittal axis of the participant. The length of the curved paths in the two sets was equal to 2 and 3 times the length of the corresponding straight paths, respectively $(B=15 \mathrm{~cm} ; \mathrm{C}=22.5$ $\mathrm{cm} ; \mathrm{E}=45 \mathrm{~cm} ; \mathrm{F}=67.5 \mathrm{~cm}$ ). Responses were given by following the $45 \mathrm{~cm}$ vertical path on the left side of the board.

Figure 3 : Longueur des trajets (sans point d'inflexion) explorés durant la phase d'encodage (Faineteau, Gentaz et Viviani, 2005). 1) Echelle 1: $\mathrm{A}=1 \times 7.5 \mathrm{~cm}, \mathrm{~B}=2 \times 7.5 \mathrm{~cm}, \mathrm{C}=3 \times$ $7.5 \mathrm{~cm}$ et $\mathrm{D}=4 \times 7.5 \mathrm{~cm}$; 2) Echelle $2: \mathrm{E}=1 \times 15 \mathrm{~cm}, \mathrm{~F}=2 \times 7.5 \mathrm{~cm}, \mathrm{G}=3 \times 7.5 \mathrm{~cm}$ et $\mathrm{H}=4$ x $7.5 \mathrm{~cm}$; 3) Echelle $3: \mathrm{I}=1 \times 22.5 \mathrm{~cm}, \mathrm{~J}=2 \times 22.5 \mathrm{~cm}, \mathrm{~K}=3 \times 22.5 \mathrm{~cm}$ et $\mathrm{L}=4 \times 22.5 \mathrm{~cm}$. 
Figure 3 : Outlay of the work plane. Each scale (S1: A, B, C, D; S2: E, F, G, H; and S3: I, J, K, L) included one straight and three half elliptic paths Faineteau, Gentaz et Viviani, 2005). The straight paths (A, E and I) were 7.5, 15 and $22.5 \mathrm{~cm}$ long, respectively, and were parallel to the sagittal axis of the participant. The length of the curved paths in the three sets was equal to two, three and four times the length of the corresponding straight paths, respectively $(B=15$ $\mathrm{cm}, \mathrm{C}=22.5 \mathrm{~cm}, \mathrm{D}=30 \mathrm{~cm}, \mathrm{~F}=30 \mathrm{~cm}, \mathrm{G}=45 \mathrm{~cm}, \mathrm{H}=60 \mathrm{~cm}, \mathrm{~J}=45 \mathrm{~cm}, \mathrm{~K}=67.5 \mathrm{~cm}, \mathrm{~L}=90 \mathrm{~cm})$. Responses were given by following the $45-\mathrm{cm}$ vertical path on the left side of the board

Figure 4 : Schéma de l'espace de travail. Chaque échelle $\left(R:\left\{R_{0}, R_{2}, R_{4}, R_{6}\right\}\right.$ et $I:\left\{I_{0}, I_{2}, I_{4}\right.$, $\left.\left.I_{6}\right\}\right)$ inclut un trajet rectiligne et trois trajets courbes. Les longueurs des trajets rectilignes $\left(\mathrm{R}_{0}\right.$ et $\mathrm{I}_{0}$ ) sont de $7.5 \mathrm{~cm}$ et de $22.5 \mathrm{~cm}$ respectivement, et sont parallèles à l'axe sagittal du sujet. Pour chaque échelle les trajets courbes sont deux fois plus longs que les trajets rectilignes correspondant et pouvaient inclure deux, quatre ou six points d'inflexions (figurés par les cercles). Les sujets donnaient leur réponse sur un trajet de $45 \mathrm{~cm}$ disposé à gauche de l'espace de travail (Faineteau, Palluel-Germain \& Gentaz, 2007).

Figure 4 : Outlay of the workplane. Each scale $\left(\mathrm{R}:\left\{\mathrm{R}_{0}, \mathrm{R}_{2}, \mathrm{R}_{4}, \mathrm{R}_{6}\right\}\right.$ et $\left.\mathrm{I}:\left\{\mathrm{I}_{0}, \mathrm{I}_{2}, \mathrm{I}_{4}, \mathrm{I}_{6}\right\}\right)$ included one straight, and three variable-curvature paths. The straight paths $\left(\mathrm{R}_{0}\right.$ and $\left.\mathrm{I}_{0}\right)$ were $7.5 \mathrm{~cm}$ and $22.5 \mathrm{~cm}$ long, respectively, and were parallel to the sagittal axis of the participant. In each set, the curved paths had either, 2, 4 or 6 inflection points, and their length was equal to 2 times the length of the corresponding straight paths. Responses were given by following the $45 \mathrm{~cm}$ vertical path on the left side of the board (Faineteau, Palluel-Germain \& Gentaz, 2007). 
Figure 5 : Schéma du dispositif experimental (Wydoodt, Gentaz \& Streri, 2006). The brasrobot à retour d'effort (Phantom 1.5) produit des forces qui peuvent être perçues par le sujet travaillant sous occlusion visuelle momentanée via son index tenu à son extrémité. Le dispositif génère ainsi un champ de force représentant un segment virtuel où tous les vecteurs sont orientés en direction de l'axe central du cylindre (flèche bleue). Les forces de traction (figure de gauche, flèche verte) ou d'opposition (figure de droite, figure rouge) sont colinéaires au segment virtuel, i.e. au mouvement d'exploration manuel. La flèche noire indique la direction du mouvement et sa vitesse (v).

Figure 5. Depiction of the experimental setup (Wydoodt, Gentaz \& Streri, 2006). The armrobot produces forces which can be felt when finger is fixed to its extremity. The virtual segment is in fact a real time computed force field of which vectors are oriented toward the central axe of a cylinder (blue arrows). Disruption forces are colinear to the segment (and the exploratory movement) and are represented by red or green arrows. Black arrow indicates the direction of the movement Left: Disruption (red arrow) is oriented in opposition to exploratory movement along the horizontal virtual rod (exp. 1 and 2). Right: Disruption (green arrow) is in addition to exploratory movement (exp. 3 and 4).

Figure 6 : Exemples de distributions de probabilités de Laplace. Elles sont toutes centrées sur le même pic $\mathrm{a}=10$, et ont des paramètres de dispersion $\mathrm{b}$ différents.

Figure 6: Examples of Laplace probabilty distributions. They are all centred on the same peak $\mathrm{a}=10$, but have different dispersion parameters $\mathrm{b}$. 
Figure 7 : Histogramme de 50 points tirés aléatoirement selon une distribution de probabilités gaussienne, superposé aux estimées de maximum de vraisemblance basées sur un modèle gaussien ou de Laplace. Les distributions de probabilités sont mises à l'échelle verticalement, pour plus de lisibilité. En abscisse, valeurs prises par les points tirés aléatoirement (unité et domaine entre 25 et 35 arbitraires); en ordonnée, effectif de chaque intervalle de l'histogramme.

Figure 7: Histogram based on 50 points drawn randomly according to a Gaussian probability distribution, superposed with the maximum likelihood Laplace and Gaussian estimates. The probability distributions are scaled vertically, for better readability. On the x-axis: values taken by the randomly drawn points (arbitrary unit and range, between 25 and 35); on the yaxis: number of points in each histogram bin." 
Figure 1

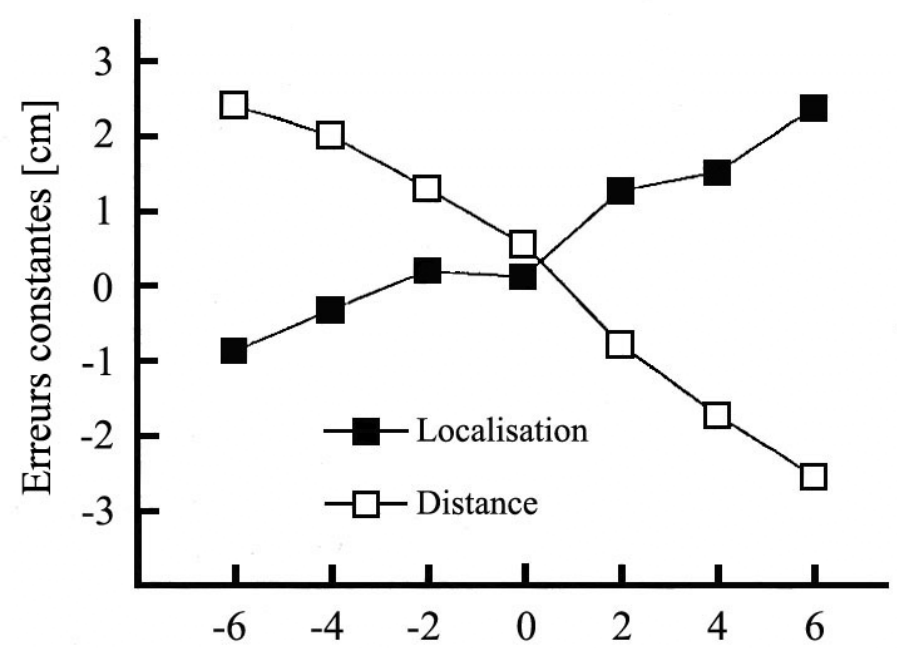

Déplacement du point de départ [cm] 
Figure 2

A

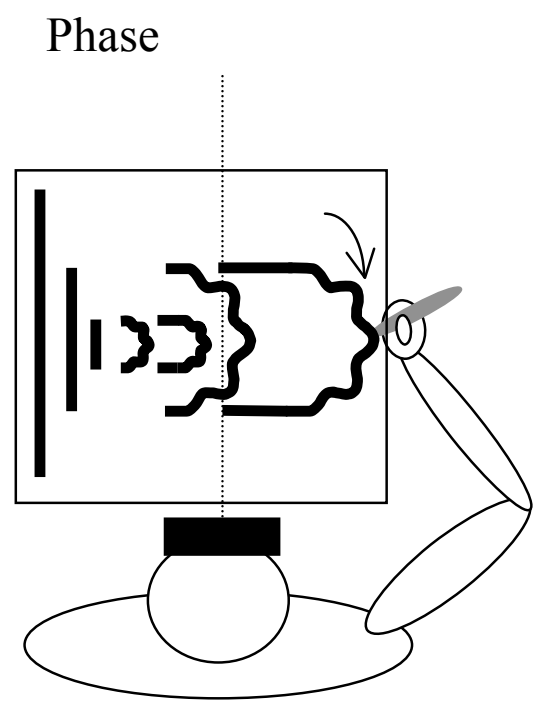

Phase de

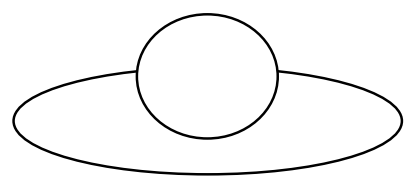

B

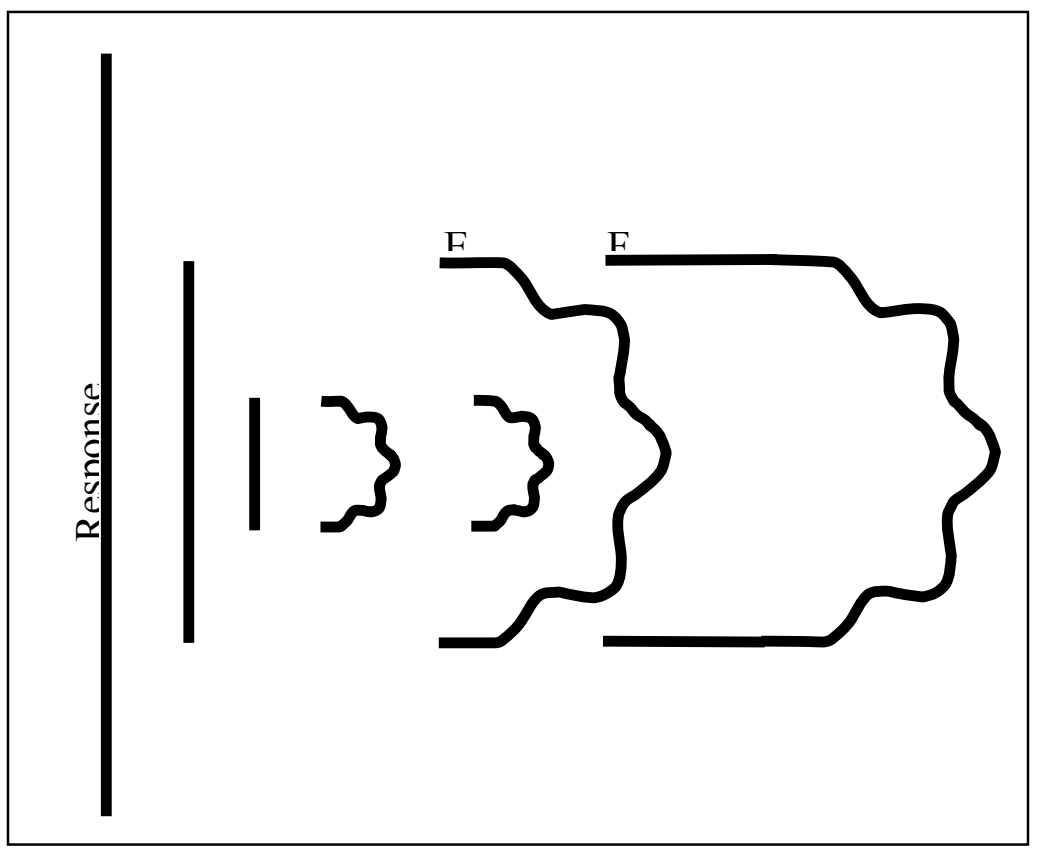


Figure 3

ÉCHELLE 1: Distance Euclidienne de 7.5

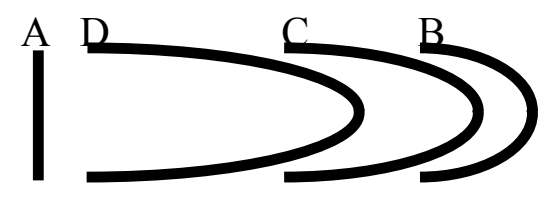

ÉCHELLE 2: Distance Euclidienne de

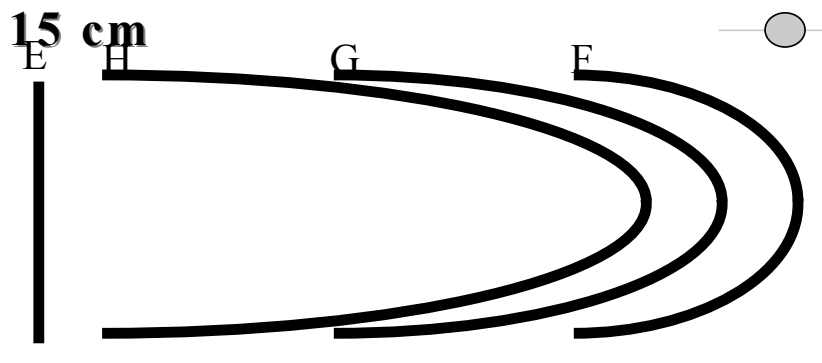

ÉCHELLE 3 : Distance Euclidienne de 22.5

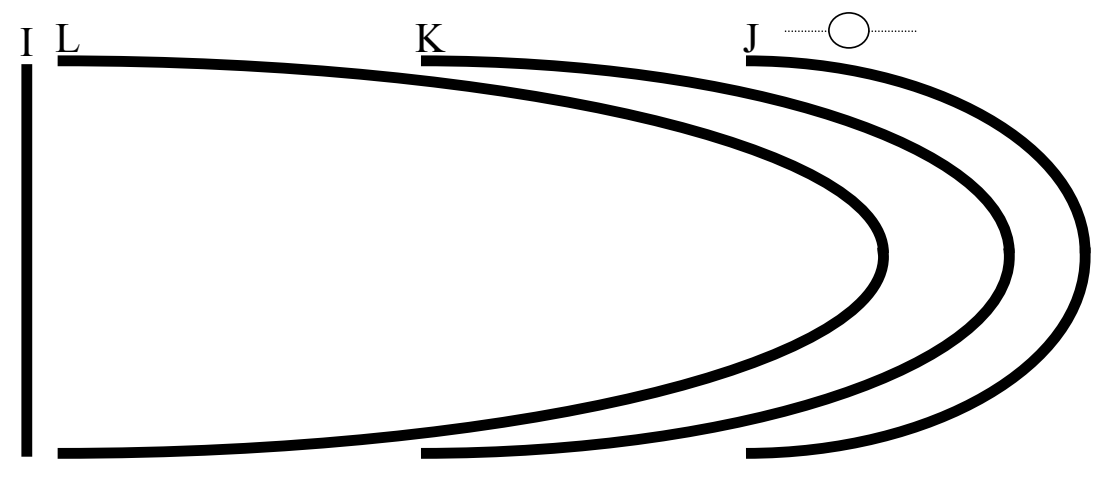


Figure 4

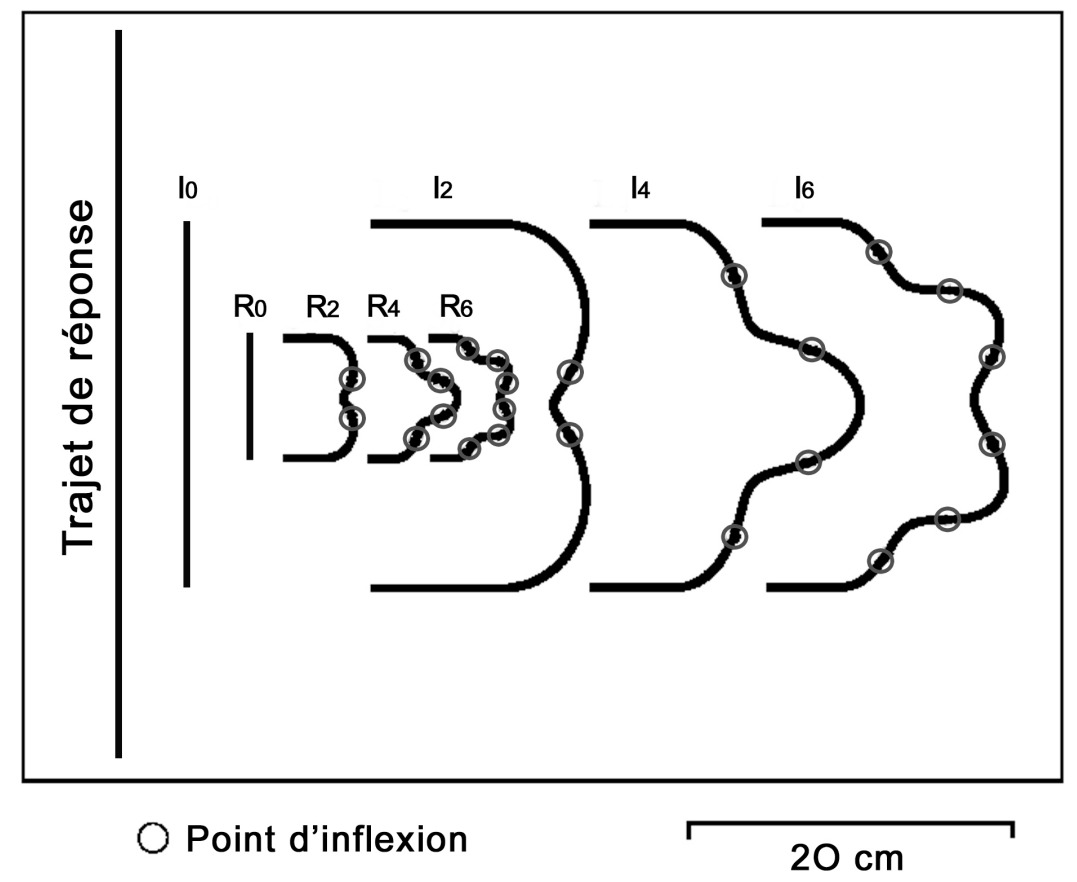


Figure 5
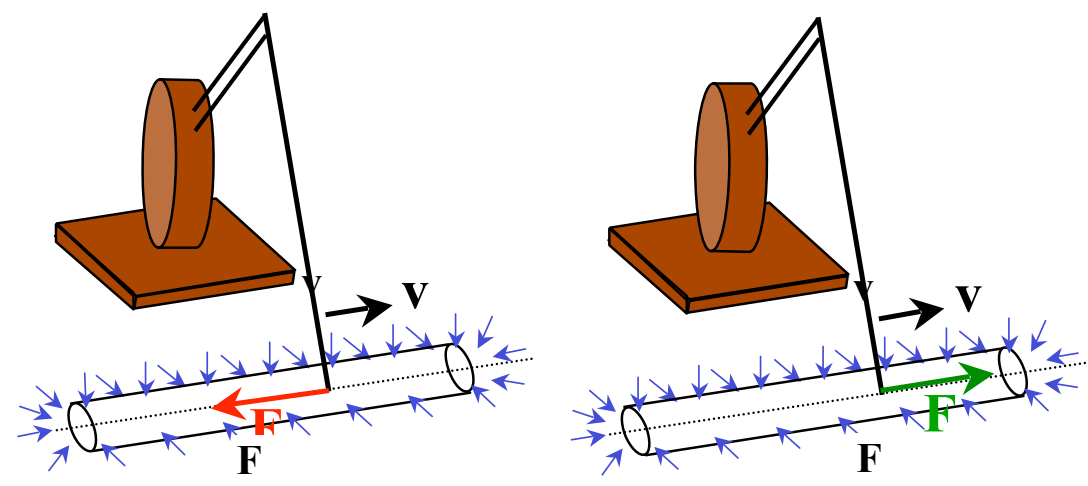
Figures 6 et 7 (cf fichier annexe en pdf) 